Article

\title{
Inventory Models for Non-Instantaneous Deteriorating Items with Expiration Dates and Imperfect Quality under Hybrid Payment Policy in the Three-Level Supply Chain
}

\author{
Jui-Jung Liao ${ }^{1}$ D, Hari Mohan Srivastava ${ }^{2,3,4,5, * D}$, Kun-Jen Chung ${ }^{6,7}$, Shih-Fang Lee ${ }^{8}$, Kuo-Nan Huang ${ }^{9}$ \\ and Shy-Der Lin 10
}

Citation: Liao, J.-J.; Srivastava, H.M.; Chung, K.-J.; Lee, S.-F.; Huang, K.-N.; Lin, S.-D. Inventory Models for Non-Instantaneous Deteriorating Items with Expiration Dates and Imperfect Quality under Hybrid Payment Policy in the Three-Level Supply Chain. Symmetry 2021, 13, 1695. https://doi.org/10.3390/ sym13091695

Academic Editors: Zhivorad Tomovski and Guangdong Tian

Received: 30 June 2021

Accepted: 31 August 2021

Published: 14 September 2021

Publisher's Note: MDPI stays neutral with regard to jurisdictional claims in published maps and institutional affiliations.

Copyright: (c) 2021 by the authors. Licensee MDPI, Basel, Switzerland. This article is an open access article distributed under the terms and conditions of the Creative Commons Attribution (CC BY) license (https:// creativecommons.org/licenses/by/ $4.0 /)$.
1 Department of Business Administration, Chihlee University of Technology, Banqiao District, New Taipei City 22050, Taiwan; liaojj@mail.chihlee.edu.tw

2 Department of Mathematics and Statistics, University of Victoria, Victoria, BC V8W 3R4, Canada

3 Department of Medical Research, China Medical University Hospital, China Medical University, Taichung 40402, Taiwan

4 Department of Mathematics and Informatics, Azerbaijan University, 71 Jeyhun Hajibeyli Street, Baku AZ1007, Azerbaijan

5 Section of Mathematics, International Telematic University Uninettuno, I-00186 Rome, Italy

6 College of Business, Chung Yuan Christian University, Chung Li 320314, Taiwan; kjchung@cycu.edu.tw

7 Department of Industrial Management, National Taiwan University of Science and Technology, Taipei 10607, Taiwan

8 Department of Applied Mathematics, Chung Yuan Christian University, Chung Li 320314, Taiwan; g10601101@cycu.edu.tw

9 Department of Industrial Management and Business Administration, St. John's University, Tamsui District, New Taipei City 25135, Taiwan; hsiaosa@mail.sju.edu.tw

10 Department of Applied Mathematics and Business Administration, Chung Yuan Christian University, Chung Li 320314, Taiwan; shyder@cycu.edu.tw

* Correspondence: harimsri@math.uvic.ca

\begin{abstract}
This article considers an inventory model for non-instantaneous deteriorating items with expiration dates, such as seasonal items, first-hand vegetables, and fruits. Interestingly, an inspection will be performed to manage the quality of the items during the state of no deterioration because it is difficult to purchase items with $100 \%$ perfection. Additionally, we assume that the upstream member has the power of controlling or influencing downstream members' decisions. That is, the supplier asks the retailer for a partial advance payment to avoid cancellation of orders and offers them a credit payment to stimulate sales; in turn, the customer must pay some cash when placing an order and pay the remainder in credit for the retailer. The goal of this article is to determine an optimal replenishment cycle and the total annual cost function, so we explore the functional properties of the total annual cost function and show that the total annual cost function is convex. Theoretical analysis of the optimal properties shows the existence and uniqueness of the optimal solution. Then, we obtain simple and easy solution procedures for the inventory system. Moreover, numerical analysis of the inventory model is conducted, and the corresponding examples are considered with a view to illustrating the application of the supply chain model that we have investigated in this article. Finally, in the concluding section, we have not only provided the motivation and the need for our usages of mathematical analytic solution procedures based upon the convexity, monotonicity (increasing and decreasing) and differentiability properties of the object function (that is, the total annual cost function), which involve some symmetry aspects of the object function, but we have also indicated the limitations and shortcomings in our investigation, which will naturally lead to some potential directions for further research on the supply chain model, which we have considered and mathematically analyzed in this article.
\end{abstract}


Keywords: inventory modeling; mathematical analytic solution procedures; economic order quantity (EOQ) model; deteriorating products; imperfect quality; hybrid payment policy; non-instantaneous deterioration; expiration date; trade credit financing; permissible delay in payments; object function (that is total annual cost function); supply chain management

\section{Introduction}

In today's competitive business environment, more than $80 \%$ of businesses offer their products on various short-term, interest-free credit terms (that is, a credit payment) with a view to stimulate sales and to reduce inventory in the United Kingdom and the United States of America. Likewise, trade credit financing is used by approximately $60 \%$ of international trade transactions, rendering it to be the second after that of banks and other financial institutions in the United States of America. Additionally, in order to avoid the risk of order cancellation and non-payment risks, the business frequently offers a partial credit period to the downstream members, who must pay a portion of the procurement amount at the time of placing an order and then receive a permissible delay on the rest of the outstanding amount (that is, a cash-credit payment). On the other hand, granting trade credit increases not only the opportunity loss, but also the default risk from the viewpoint of the business, so the powerful businesses may ask the downstream members to prepay the entire or a fraction of the procurement amount before the delivery to mitigate interest loss and default risks (that is, an advance payment). For example, insurance companies generally require an advance payment in order to extend coverage to the insured party.

In existing literatures, Zhang [1] proposed an advance payment plan because it may save time and money for a customer to prepay, for example 80.00 USD for 4 months of water bills, instead of paying 20.00 USD each month for 4 months. All the above mentioned payment types can be combined such that, for example, the supplier demands the retailer to prepay 5 to $10 \%$ of the total procurement cost as a good-faith deposit when both sign a contract of agreement to install some item(s). Upon delivery of the item(s), a cashon-delivery payment to cover the supplier's cost of the item(s) is then required. In this contractual arrangement, the retailer will pay the remainder of the total cost after the work is completed.

In reality, the deteriorating items have a maximum lifetime due to their physical nature and must be disposed of after the expiration date, due to the fact that consumers evaluate the freshness of a deteriorating item by checking its expiration date before making a purchase, and the willingness to purchase a deteriorating item decreases throughout its shelf-life. Furthermore, the expiration date is the most important factor that is time-bound and plays an important role in developing the inventory model. In practice, most of the products maintain their quality or original situation over a span; that is, during this span, deterioration does not occur, and then they begin to deteriorate in the next period. It is observed that foodstuffs, first-hand vegetables, and fruits have a short span during which fresh quality is maintained and there is almost no spoilage. These processes are defined as the non-instantaneous deterioration of the product.

The quality of the products is considered to be another direct factor to affect a consumer's purchase decision as well. Furthermore, this article assumes that the retailer receives the items with a time-varying deterioration rate depending on its expiration date, such as seasonal products, and that an inspection will occur during the state of no deterioration in order to manage the quality of the products. By performing the screening process, the retailer detects the imperfect items and throws them out.

In this article, we first establish an inventory model for non-instantaneous deteriorating items with expiration dates and imperfect quality in which we assume a 100\% screening process to identify imperfect items. We then consider that the supplier asks the retailer to prepay a fraction of the procurement cost when signing a contract to buy products, to pay another fraction of the procurement cost in cash upon receiving the ordered quantity, and 
to receive a short-term interest-free credit term on the remaining procurement cost (that is, an advance-cash-credit payment). Likewise, the retailer gives the customer the opportunity to pay a fraction of the procurement cost after delivery of the ordered items and then to pay the remaining procurement cost at a later date without any additional charges to reduce the risk of cancellations of the order from customers (that is, a cash-credit payment). It is worth mentioning that, by the usage of the mathematical analytic solution procedures, the present article shows that the total annual cost function is convex by exploring the functional properties of the total annual cost function such as, for example, the continuity, convexity, monotonicity (increasing and decreasing), and differentiability properties, whereby one can also see the symmetry aspects of the total annual const function. Furthermore, by applying the mathematical analytic solution procedures again, we prove that the retailer's optimal replenishment cycle not only exists, but it also is unique. With a view to illustrating and validating the proposed inventory model, we have considered numerical examples involving different fixed markup rates. Finally, in the concluding section of this article (Section 6), we have briefly discussed the limitations and shortcomings of this investigation in that we have concentrated upon the inventory system without shortage and that it can affect the supply chain from the producer to the retailer. Furthermore, this model has the potential to be extended to incorporate inflation and quantity discount effects, different demand forms such as credit-linked promotion-dependent demand and other issues under the system with shortages. Additionally, this article has considered the deterministic situation, so considering the stochastic situation, such as stochastic demand, can be another future research direction on the subject of this article.

\section{Literature Review}

There is a large volume of published studies concerning the inventory models with cash payments, credit payments, or advance payments, such as those that we have reviewed or cited below.

In these literatures, Taleizadeh et al. [2] established an economic order quantity (EOQ) model with partial backordering in which the supplier asks the retailer to pay a fraction of the purchasing cost in advance and allows them to divide the prepayment into multiple equal installments during a lead time. Taleizadeh [3] extended the inventory model of Taleizadeh et al. [2] to the cases of deteriorating items with and without shortages. Taleizadeh [4] used an advance-cash-payment plan to develop an EOQ model for an evaporating item with partial backordering for a real case study of a gasoline station. Zhang et al. [5] developed an inventory model under advance payment, which includes all payments in advance and partially advanced-partially delayed payment plans. Eck et al. [6] explored the role of cash-in-advance financing for export decisions in firms. Lashgari et al. [7] considered an EOQ model with hybrid partial payment, such as upstream partial prepayment and downstream partial delayed payment without shortage, with full backordering, or with partial backordering. Tavakoli and Taleizadeh [8] gave a lot-sizing model for decaying item for the retailer to pay all the purchasing cost in advance with no shortage or with full backordering shortage or partial lost sale. Heydari et al. [9] assumed the demand is stochastic and credit-dependent under the two-level trade credit, then they found the optimal ordering quantity and the length of the credit period. Feng and Chan [10] expanded the two-level trade credit to include joint pricing and production decisions for new products with pronounced learning-by-doing phenomenon. Much of the current research attention has been directed towards trade-credit inventory models for deteriorating items with its own expiration date. For example, Chen and Teng [11] established an inventory model for deteriorating items under two-level trade credit by discounted cash flow analysis in which the deterioration rate is non-decreasing over time and near 100 percent particularly close to its expiration date. Then, they demonstrated that the retailer's optimal credit period and cycle time not only exist, but are also unique. Mahata [12] discussed an EOQ model for deteriorating items having fixed lifetime under two-level trade credit. He showed that the retailer's optimal replenishment cycle time not only exists but is also 
unique. Wu et al. [13] examined an inventory model with expiration date dependent deterioration under an advance-cash-credit payment scheme to find the optimal replenishment cycle time and the fraction of no shortages such that the total profit is maximized. Moreover, some related recent articles are those by, for example, Zia and Taleizadeh [14], Wu et al. [15], Chen et al. [16], Teng et al. [17], Diabat et al. [18], Feng et al. [19], Mahata and De [20], Tiwari et al. [21], Taleizadeh et al. [22], Li et al. [23,24], Taleizadeh [25], Krommyda et al. [26], Tsao et al. [27], Mashud et al. ([28,29]), AlArjani et al. [30], and Hou et al. [31].

There is a large volume of published studies describing the inventory models for non-instantaneous deteriorating items. Udayakumar and Geetha [32] considered time, value of money, and the effect of inflation to develop an economic-ordering policy for non-instantaneous deteriorating items over a finite time horizon in which the demand is a deterministic function of selling price and advertisement cost. They found the optimal length of replenishment and the optimal order quantity. Lashgari et al. [33] presented an EOQ model for non-instantaneous deteriorating items under an advance-delay payment when shortages are allowed in a partial form. They found the optimal order and shortage quantities to minimize the retailer's total inventory cost function. Udayakumar and Geetha [34] developed an EOQ model for non-instantaneous deteriorating items with capacity constraint under a trade credit policy. They found the optimal replenishment cycle time and order quantity to minimize the total inventory cost. Babangida and Baraya [35] showed an inventory model for non-instantaneous deteriorating items with two-phase demand rates, capacity constraint and complete backlogged under trade credit policy. They provided the necessary and sufficient conditions for the existence and uniqueness of solutions. Soni and Suthar [36] revealed an inventory model for non-instantaneous deteriorating items with partial backlogging; they considered that the demand rate has a negative and positive exponential effect of price and promotional effort, respectively, while the item is not in a state of deterioration and then found the joint optimal pricing and replenishment policy for the non-instantaneous deteriorating items. Cenk Çalışkan [37] deals with the inventory model for deteriorating items in which the opportunity cost is based on compound interest, and backorders are allowed. The article determines a near-optimal and intuitive closed-form solution, which is simple to the practitioners. Under a variety of practical conditions, some researchers have considered the above items, such as Tiwari et al. [38], Tsao [39], Geetha and Udayakumar [40], Jaggi et al. [41], Maihami et al. [42], Mashud et al. [43], Bounkhel et al. [44], and Udayakumar et al. [45].

Given that it is worthwhile studying the effect of defective items on inventory problems, numerous researchers, such as Khanna et al. [46], have developed inventory models for deteriorating imperfect quality items with allowable shortages and permissible delays in payments. Zhou et al. [47] found a synergy economic order quantity model, in which the concepts of imperfect quality, inspection error, and shortages with trade credit are considered. They found the annual profit function is concave and obtained the closed form optimal solution to the model. Datta [48] proposed a production-inventory model with defective items. The model incorporates additional investment opportunity on quality improvement for reducing the proportion of defective products. Taleizadeh et al. [49] developed an imperfect EPQ model with upstream trade credit periods linked to raw material order quantity and downstream trade credit periods. Pal and Mahapatra [50] developed an inventory model with imperfect products for a three-level supply chain, and three different ways of dealing with defective products were investigated in their model. Khakzad and Gholamian [51] investigated the effect of inspection time on the deterioration rate; they showed the convexity of the model and illustrated the uniqueness of the solution. Taleizadeh et al. [52] revealed an EOQ inventory model with imperfect items and partial backordering. They assumed a percent of the products in a lot is imperfect and the imperfect items are replenished by perfect ones at a higher cost. The objective is to obtain the optimal value of the length period and the percent of period duration in which the inventory level is positive. Some imperfect production models with trade credit have been studied, in recent years, by (among others) Wang et al. [53], Alamri 
et al. [54], Palanivel and Uthayakumar [55], Aghili and Hoseinabadi [56], Tsao et al. [57,58], Khanna et al. [59], Liao et al. [60], Kazemi et al. [61], Liao et al. [62], Mashud et al. [63], and Srivastava et al. [64].

We remark, in passing to the next section, that the mathematical analytic solution procedures, which we have used in the mathematical analysis and discussions of the inventory and supply chain models considered in this article, are full of elaborate usages of the intricate techniques of calculus in determining the continuity, convexity, monotonicity (increasing or decreasing), and differentiability properties of the object functions (that is, the total optimal cost functions). We have stated our main results of this investigation in the form of five theorems (Theorems 1 to 5), which we have proved by appealing also to two Lemmas (Lemma 1 and Lemma 2). For the sake of brevity and compact presentation, the proof of Lemma 2 has been given in the Appendix A instead of the main text. It is quite natural to expect such a format and style in a mathematically oriented article. Furthermore, as we have already mentioned, for the accuracy, completeness, and safe applicability of the results and discussions presented in this article, the usage of the mathematical analytic solution procedures, which are based upon the elaborate and intricate techniques of calculus, is essential here.

\section{Mathematical Formulation of the Supply Chain Model and Its Analysis}

Based on the above assumptions, the inventory level drops at the demand rate and the defective rate during the time interval $\left[0, t_{d}\right]$. Then, the inventory level drops to zero due to the demand and the deterioration with the expiration dates during the time interval $\left[t_{d}, T\right]$. Furthermore, the variations in the inventory level with respect to time $t$ can be expressed below.

The differential equation representing the inventory status during the time interval $t \in\left[0, t_{s}\right], t_{s}=\frac{y}{x}$, is given by

$$
\frac{d I_{1}(t)}{d t}=-D
$$

where $t$ is restricted, as in Equation (2). Under the condition $I_{1}(0)=y$, by solving Equation (1), we obtain

$$
I_{1}(t)=y-D t, 0<t \leq t_{s}
$$

In the second interval $\left[t_{s}, t_{d}\right]$, the differential equation represents the inventory status:

$$
\frac{d I_{2}(t)}{d t}=-D, t_{s} \leq t \leq t_{d}
$$

Under the condition $I_{2}\left(t_{d}\right)=(1-p) y-D t_{d}$, Equation (3) yields

$$
I_{2}(t)=(1-p) y-D t, t_{s} \leq t \leq t_{d}
$$

During the third interval $\left[t_{d}, T\right]$, the change in the inventory level is represented by the following differential equation:

$$
\frac{d I_{3}(t)}{d t}+\theta(t) \cdot I_{3}(t)=-D, t_{d} \leq t \leq T
$$

Under the condition $I_{3}(T)=0$, the solution of Equation (5) is given by

$$
I_{3}(t)=D(1+m-t) \cdot \ln \left(\frac{1+m-t}{1+m-T}\right), t_{d} \leq t \leq T
$$

Making use of the continuity property of $I_{2}\left(t_{d}\right)=I_{3}\left(t_{d}\right)$, it follows from Equations (4) and (6) that

$$
(1-p) y-D t_{d}=D\left(1+m-t_{d}\right) \ln \left(\frac{1+m-t_{d}}{1+m-T}\right)
$$


which implies that the order quantity is given by

$$
y=\frac{D}{(1-p)}\left[t_{d}+\left(1+m-t_{d}\right) \cdot \ln \left(\frac{1+m-t_{d}}{1+m-T}\right)\right]
$$

Substituting Equation (8) into Equations (2) and (4), we get

$$
I_{1}(t)=\frac{D}{(1-p)}\left[t_{d}+\left(1+m-t_{d}\right) \cdot \ln \left(\frac{1+m-t_{d}}{1+m-T}\right)-(1-p) t\right]
$$

and

$$
I_{2}(t)=D\left[t_{d}+\left(1+m-t_{d}\right) \cdot \ln \left(\frac{1+m-t_{d}}{1+m-T}\right)-t\right]
$$

Additionally, this article focuses on $t_{s} \leq t_{d}$, so we have $T \leq R^{*}$ if and only if $t_{s} \leq t_{d}$. Here,

$$
R^{*}=(1+m)-\left(1+m-t_{d}\right) \cdot e^{-\frac{[(1-p) x-D] t_{d}}{D\left(1+m-t_{d}\right)}}
$$

We now calculate the annual total relevant cost which results from the following components:

1. $\quad$ Order cost $=\frac{O}{T}$

2. The holding cost (excluding interest charges) after receiving $y$ units at time 0 is given below:

Case 1. When $0<T<t_{d}$,

The holding cost

$$
=\frac{h}{T}\left[\int_{0}^{\frac{y}{x}}(y-D t) d t+\int_{\frac{y}{x}}^{T} D(T-t) d t\right]=\frac{h D}{(1-p)}\left[\frac{(1-p) T}{2}+\frac{p D T}{x(1-p)}\right]
$$

Case 2. When $t_{d} \leq T$

The holding cost $=\frac{h}{T}\left\{\int_{0}^{\frac{y}{x}} I_{1}(t) d t+\int_{\frac{y}{x}}^{t_{d}} I_{2}(t) d t+\int_{t_{d}}^{T} I_{3}(t) d t\right\}$

$$
\begin{aligned}
& =\frac{h}{T}\left\{\frac{D}{2} t_{d}^{2}+D\left(1+m-t_{d}\right) \cdot t_{d} \ln \left(\frac{1+m-t_{d}}{1+m-T}\right)+\frac{p D^{2}}{x(1-p)^{2}}\right. \\
& \times\left[t_{d}+\left(1+m-t_{d}\right) \cdot \ln \left(\frac{1+m-t_{d}}{1+m-T}\right)\right]^{2}+\frac{D}{2}\left(1+m-t_{d}\right)^{2} \cdot \ln \left(\frac{1+m-t_{d}}{1+m-T}\right) \\
& \left.+\frac{D}{4}(1+m-T)^{2}-\frac{D}{4}\left(1+m-t_{d}\right)^{2}\right\}
\end{aligned}
$$

3. The procurement cost per replenishment cycle is:

Case 1. When $0<T<t_{d}$

The procurement $\cos t=\frac{c y}{T}=\frac{c D}{(1-p)}$

Case 2. When $t_{d} \leq T$

The procurement cost $=\frac{c y}{T}=\frac{c D}{(1-p) T}\left[t_{d}+\left(1+m-t_{d}\right) \cdot \ln \left(\frac{1+m-t_{d}}{1+m-T}\right)\right]$

4. The screening cost per replenishment cycle is

Case 1 . When $0<T<t_{d}$

The screening cost $=\frac{s y}{T}=\frac{s D}{(1-p)}$

Case 2. When $t_{d} \leq T$

The screening cost $=\frac{s y}{T}=\frac{s D}{(1-p) T}\left[t_{d}+\left(1+m-t_{d}\right) \cdot \ln \left(\frac{1+m-t_{d}}{1+m-T}\right)\right]$

5. The cost of deteriorated units

Case 1 . When $0<T<t_{d}$

The cost of deteriorated units is zero.

Case 2. When $t_{d} \leq T$

The cost of deteriorated units $=\frac{c}{T}[(1-p) y-D T]$

$$
=\frac{c D}{T}\left[t_{d}+\left(1+m-t_{d}\right) \cdot \ln \left(\frac{1+m-t_{d}}{1+m-T}\right)-T\right]
$$


6. The interest charged for advance payment per replenishment cycle is

$$
\begin{aligned}
& \frac{c I_{k} D T}{T}\left(\int_{-L}^{N} \alpha d t\right)+\frac{c I_{k} D}{T} \int_{N}^{T+N} \alpha(T+N-t) t \\
& =\frac{\alpha c I_{k} D T}{T}(N+L)+\frac{\alpha c I_{k} D T^{2}}{2 T}
\end{aligned}
$$

7. The interest charged for cash payment per replenishment cycle is

$$
\frac{c I_{k} D T}{T}\left(\int_{0}^{N} \beta d t\right)+\frac{c I_{k} D}{T} \int_{N}^{T+N} \beta(T+N-t) d t=\frac{\beta c I_{k} D T N}{T}+\frac{\beta c I_{k} D T^{2}}{2 T}
$$

8. The interest charged for credit payment per replenishment is

Case 1 . When $N \leq M$ and $M \leq T \leq T+N$

The interest charged for credit payment

$$
\begin{gathered}
=\frac{\tau c D I_{k}}{T}\left\{\rho\left[\int_{M}^{T+N}(T+N-t) d t\right]+(1-\rho)\left[\int_{M}^{T}(T-t) d t\right\}\right. \\
=\frac{\tau c D I_{k}}{2 T}\left\{\rho(T+N-M)^{2}+(1-\rho)(T-M)^{2}\right\}
\end{gathered}
$$

Case 2. When $N \leq M$ and $T \leq M \leq T+N$

The interest charged for credit payment

$$
=\frac{\tau c D I_{k}}{T}\left[\rho \int_{M}^{T+N}(T+N-t) d t\right]=\frac{\tau c D I_{k}}{2 T}\left[\rho(T+N-M)^{2}\right]
$$

Case 3. When $N \leq M$ and $T+N \leq M$

The interest charged for credit payment is zero.

Case 4 . When $N>M$ and $M \leq T$

The interest charged for credit payment

$$
\begin{gathered}
=\frac{\tau c D I_{k}}{T}\left\{\rho\left[\int_{M}^{N} T d t+\int_{N}^{T+N}(T+N-t) d t\right]+(1-\rho)\left[\int_{M}^{T}(T-t) d t\right\}\right. \\
=\frac{\tau c D I_{k}}{2 T}\left\{\rho\left[T^{2}+2(N-M) T\right]+(1-\rho)(T-M)^{2}\right\}
\end{gathered}
$$

Case 5. When $N>M$ and $M \geq T$

The interest charged for credit payment

$$
\begin{gathered}
=\frac{\tau c D I_{k}}{T}\left\{\rho\left[\int_{M}^{N} T d t+\int_{N}^{T+N}(T+N-t) d t\right]\right\} \\
=\frac{\tau c D I_{k}}{2 T}\left\{\rho\left[T^{2}+2 T(N-M)\right]\right\}
\end{gathered}
$$

9. The interest earned for credit payment per replenishment is

Case 1. When $N \leq M$ and $M \leq T \leq T+N$

The interest earned for credit payment

$$
=\frac{\tau v D I_{e}}{T}\left\{\rho \int_{N}^{M}(t-N) d t+(1-\rho) \int_{0}^{M} t d t\right\}=\frac{\tau v D I_{e}}{2 T}\left[\rho(M-N)^{2}+(1-\rho) M^{2}\right]
$$

Case 2. When $N \leq M$ and $T \leq M \leq T+N$

The interest earned for credit payment

$$
=\frac{\tau v D I_{e}}{T}\left\{\rho \int_{N}^{M}(t-N) d t+(1-\rho)\left[\int_{0}^{T} t d t+\int_{T}^{M} T d t\right]\right\}
$$




$$
=\frac{\tau v D I_{e}}{2 T}\left\{\rho(M-N)^{2}+(1-\rho)\left[T^{2}+2 T(M-T)\right]\right\}
$$

Case 3. When $N \leq M$ and $T+N \leq M$

The interest earned for credit payment

$$
\begin{gathered}
=\frac{\tau v D I_{e}}{T}\left\{\rho\left[\int_{N}^{T+N}(t-N) d t+\int_{T+N}^{M} T d t\right]+(1-\rho)\left[\int_{0}^{T} t d t+\int_{T}^{M} T d t\right]\right\} \\
=\frac{\tau v D I_{e}}{2 T}\left\{\rho\left[T^{2}+2 T(M-T-N)\right]+(1-\rho)\left[T^{2}+2 T(M-T)\right]\right\}
\end{gathered}
$$

Case 4. When $N>M$ and $M \leq T$

$=\frac{\tau v D I_{e}}{T}\left[(1-\rho) \int_{0}^{M} t d t\right]=\frac{\tau v D I_{e}}{2 T}\left[(1-\rho) M^{2}\right]$

Case 5. When $N>M$ and $N \geq T$

$=\frac{\tau v D I_{e}}{T}\left\{(1-\rho)\left[\int_{0}^{T} t d t+\int_{T}^{M} T d t\right]\right\}=\frac{\tau v D I_{e}}{T}\left\{(1-\rho)\left[T^{2}+2 T(M-T)\right]\right\}$

Finally, the total annual relevant cost $T C(T)$ is obtained as follows:

$T C(T)=$ ordering cost + stock holding cost (excluding interest charges) + procurement cost + screening cost + deterioration cost + interest charged - interest earned.

Furthermore, we obtain the following cases:

Case I. Suppose that $N \leq M$

Case (I-1). Suppose that $t_{d}<M-N<M$

$$
T C(T)=\left\{\begin{array}{ccc}
T C_{1}(T) & \text { if } & 0<T<t_{d} \\
T C_{2}(T) & \text { if } & t_{d} \leq T<M-N \\
T C_{3}(T) & \text { if } & M-N \leq T<M \\
T C_{4}(T) & \text { if } & M \leq T \leq R^{*}
\end{array}\right.
$$

where

$$
\begin{aligned}
& T C_{1}(T)=\frac{o}{T}+\frac{h D}{(1-p)}\left[\frac{(1-p) T}{2}+\frac{p D T}{x(1-p)}\right]+\frac{(c+s) D}{1-p}+\frac{c I_{k} D T}{T}[\alpha(N+L)+\beta N] \\
& +\frac{c_{L} D T^{2}}{2 T}(\alpha+\beta)-\frac{\tau \nu D I_{e}}{2 T}\left\{\rho\left[T^{2}+2 T(M-T-N)\right]+(1-\rho)\left[T^{2}+2 T(M-T)\right]\right\} \\
& T C_{2}(T)=\frac{o}{T}+\frac{h}{T}\left\{\frac{D}{2} t_{d}^{2}+D\left(1+m-t_{d}\right) \cdot t_{d} \cdot>\ln \left(\frac{1+m-t_{d}}{1+m-T}\right)+\frac{p D^{2}}{x(1-p)^{2}}\left[t_{d}+\left(1+m-t_{d}\right)\right.\right. \\
& \left.\left.\times \ln \left(\frac{1+m-t_{d}}{1+m-T}\right)\right]^{2}+\frac{D}{2}\left(1+m-t_{d}\right)^{2} \cdot \ln \left(\frac{1+m-t_{d}}{1+m-T}\right)+\frac{D}{4}(1+m-T)^{2}-\frac{D}{4}\left(1+m-t_{d}\right)^{2}\right\} \\
& +\frac{(c+s) D}{(1-p) T}\left[t_{d}+\left(1+m-t_{d}\right) \cdot \ln \left(\frac{1+m-t_{d}}{1+m-T}\right)\right]+\frac{c D}{T}\left[t_{d}+\left(1+m-t_{d}\right) \cdot \ln \left(\frac{1+m-t_{d}}{1+m-T}\right)-T\right] \\
& +\frac{c I_{k} D T}{T}[\alpha(N+L)+\beta N]+\frac{c I_{k} D T^{2}}{2 T}(\alpha+\beta)-\frac{\tau v D I_{e}}{2 T}\left\{\rho\left[T^{2}+2 T(M-T-N)\right]\right. \\
& \left.+(1-\rho)\left[T^{2}+2 T(M-T)\right]\right\} \\
& T C_{3}(T)=\frac{o}{T}+\frac{h}{T}\left\{\frac{D}{2} t_{d}^{2}+D\left(1+m-t_{d}\right) \cdot t_{d} \cdot \ln \left(\frac{1+m-t_{d}}{1+m-T}\right)+\frac{p D^{2}}{x(1-p)^{2}}\left[t_{d}+\left(1+m-t_{d}\right)\right.\right. \\
& \left.\left.\times \ln \left(\frac{1+m-t_{d}}{1+m-T}\right)\right]^{2}+\frac{D}{2}\left(1+m-t_{d}\right)^{2} \cdot \ln \left(\frac{1+m-t_{d}}{1+m-T}\right)+\frac{D}{4}(1+m-T)^{2}-\frac{D}{4}\left(1+m-t_{d}\right)^{2}\right\} \\
& +\frac{(c+s) D}{(1-p) T}\left[t_{d}+\left(1+m-t_{d}\right) \cdot \ln \left(\frac{1+m-t_{d}}{1+m-T}\right)\right]+\frac{c D}{T}\left[t_{d}+\left(1+m-t_{d}\right) \cdot \ln \left(\frac{1+m-t_{d}}{1+m-T}\right)-T\right] \\
& +\frac{c I_{K} D T}{T}[\alpha(N+L)+\beta N]+\frac{c I_{k} D T^{2}}{2 T}(\alpha+\beta)+\frac{\tau c D I_{k}}{2 T}\left[\rho(T+N-M)^{2}\right] \\
& -\frac{\tau v D I_{e}}{2 T}\left\{\rho(M-N)^{2}+(1-\rho)\left[T^{2}+2 T(M-T)\right]\right\} \\
& T C_{4}(T)=\frac{o}{T}+\frac{h}{T}\left\{\frac{D}{2} t_{d}^{2}+D\left(1+m-t_{d}\right) \cdot t_{d} \cdot \ln \left(\frac{1+m-t_{d}}{1+m-T}\right)+\frac{p D^{2}}{x(1-p)^{2}}\left[t_{d}+\left(1+m-t_{d}\right)\right.\right. \\
& \left.\left.\times \ln \left(\frac{1+m-t_{d}}{1+m-T}\right)\right]^{2}+\frac{D}{2}\left(1+m-t_{d}\right)^{2} \cdot \ln \left(\frac{1+m-t_{d}}{1+m-T}\right)+\frac{D}{4}(1+m-T)^{2}-\frac{D}{4}\left(1+m-t_{d}\right)^{2}\right\} \\
& +\frac{(c+s) D}{(1-p) T}\left[t_{d}+\left(1+m-t_{d}\right) \cdot \ln \left(\frac{1+m-t_{d}}{1+m-T}\right)\right]+\frac{c D}{T}\left[t_{d}+\left(1+m-t_{d}\right) \cdot \ln \left(\frac{1+m-t_{d}}{1+m-T}\right)-T\right] \\
& +\frac{c c_{k} T T}{T}[\alpha(N+L)+\beta N]+\frac{c_{k} D T^{2}}{2 T}(\alpha+\beta)+\frac{\tau c D I_{k}}{2 T}\left[\rho(T+N-M)^{2}\right. \\
& \left.+(1-\rho)(T-M)^{2}\right]-\frac{\tau V D I_{e}}{2 T}\left[\rho(M-N)^{2}+(1-\rho) M^{2}\right]
\end{aligned}
$$

Since $T C_{1}\left(t_{d}\right)=T C_{2}\left(t_{d}\right), T C_{2}(M-N)=T C_{3}(M-N)$ and $T C_{3}(M)=T C_{4}(M)$, $T C(T)$ is continuous and well-defined on $T>0$. 
Case (I-2). Suppose that $M-N<t_{d}<M$

$$
T C(T)=\left\{\begin{array}{ccc}
T C_{1}(T) & \text { if } & 0<T<M-N \\
T C_{5}(T) & \text { if } & M-N \leq T<t_{d} \\
T C_{3}(T) & \text { if } & t_{d} \leq T<M \\
T C_{4}(T) & \text { if } & M \leq T \leq R^{*}
\end{array}\right.
$$

where

$$
\begin{aligned}
T C_{5}(T) & =\frac{o}{T}+\frac{h D}{(1-p)}\left[\frac{(1-p) T}{2}+\frac{p D T}{x(1-p)}\right]+\frac{(c+s) D}{1-p}+\frac{c I_{k} D T}{T}[\alpha(N+L)+\beta N] \\
& +\frac{c I_{k} D T^{2}}{2 T}(\alpha+\beta)+\frac{\tau c D I_{k}}{2 T}\left[\rho(T+N-M)^{2}\right]-\frac{\tau v D I_{e}}{2 T}\left\{\rho(M-N)^{2}\right. \\
& \left.+(1-\rho)\left[T^{2}+2 T(M-T)\right]\right\}
\end{aligned}
$$

Since $T C_{1}(M-N)=T C_{5}(M-N), T C_{5}\left(t_{d}\right)=T C_{3}\left(t_{d}\right)$ and $T C_{3}(M)=T C_{4}(M)$, $T C(T)$ is continuous and well-defined on $T>0$.

Case (I-3). Suppose that $M-N<M<t_{d}$

$$
T C(T)=\left\{\begin{array}{ccc}
T C_{1}(T) & \text { if } & 0<T<M-N \\
T C_{5}(T) & \text { if } & M-N \leq T<M \\
T C_{6}(T) & \text { if } & M \leq T<t_{d} \\
T C_{4}(T) & \text { if } & t_{d} \leq T \leq R^{*}
\end{array}\right.
$$

where

$$
\begin{aligned}
T C_{6}(T) & =\frac{o}{T}+\frac{h D}{(1-p)}\left[\frac{(1-p) T}{2}+\frac{p D T}{x(1-p)}\right]+\frac{(c+s) D}{1-p}+\frac{c I_{k} D T}{T}[\alpha(N+L)+\beta N] \\
& +\frac{c I_{k} D T^{2}}{2 T}(\alpha+\beta)+\frac{\tau c D I_{k}}{2 T}\left[\rho(T+N-M)^{2}+(1-\rho)(T-M)^{2}\right] \\
& -\frac{\tau v D I_{e}}{2 T}\left[\rho(M-N)^{2}+(1-\rho) M^{2}\right]
\end{aligned}
$$

Since $T C_{1}(M-N)=T C_{5}(M-N), T C_{5}(M)=T C_{6}(M)$ and $T C_{6}\left(t_{d}\right)=T C_{4}\left(t_{d}\right)$, $T C(T)$ is continuous and well-defined on $T>0$.

Case II. Suppose that $N>M$

Case (II-1). Suppose that $t_{d}<M$

$$
T C(T)=\left\{\begin{array}{ccc}
T C_{7}(T) & \text { if } & 0<T<t_{d} \\
T C_{8}(T) & \text { if } & t_{d} \leq T<M \\
T C_{9}(T) & \text { if } & M \leq T \leq R^{*}
\end{array}\right.
$$

where

$$
\begin{aligned}
T C_{7}(T) & =\frac{o}{T}+\frac{h D}{(1-p)}\left[\frac{(1-p) T}{2}+\frac{p D T}{x(1-p)}\right]+\frac{(c+s) D}{1-p}+\frac{c I_{k} D T}{T}[\alpha(N+L)+\beta N] \\
& +\frac{c I_{k} D T^{2}}{2 T}(\alpha+\beta)+\frac{\tau c D I_{k}}{2 T}\left\{\rho\left[T^{2}+2 T(N-M)\right]\right\} \\
& -\frac{\tau v D I_{e}}{2 T}\left\{(1-\rho)\left[T^{2}+2 T(M-T)\right]\right\} \\
T C_{8}(T)= & \frac{o}{T}+\frac{h}{T}\left\{\frac{D}{2} t_{d}^{2}+D\left(1+m-t_{d}\right) \cdot t_{d} \cdot \ln \left(\frac{1+m-t_{d}}{1+m-T}\right)+\frac{p D^{2}}{x(1-p)^{2}}\left[t_{d}+\left(1+m-t_{d}\right)\right.\right. \\
& \left.\left.\times \ln \left(\frac{1+m-t_{d}}{1+m-T}\right)\right]^{2}+\frac{D}{2}\left(1+m-t_{d}\right)^{2} \cdot \ln \left(\frac{1+m-t_{d}}{1+m-T}\right)+\frac{D}{4}(1+m-T)^{2}-\frac{D}{4}\left(1+m-t_{d}\right)^{2}\right\} \\
& +\frac{(c+s) D}{(1-p) T}\left[t_{d}+\left(1+m-t_{d}\right) \cdot \ln \left(\frac{1+m-t_{d}}{1+m}\right)\right]+\frac{c D}{T}\left[t_{d}+\left(1+m-t_{d}\right) \cdot \ln \left(\frac{1+m-t_{d}}{1+m-T}\right)-T\right] \\
& +\frac{c_{k} k T}{\ln T}[\alpha(N+L)+\beta N]+\frac{c l_{k} D T}{2 T}(\alpha+\beta)+\frac{\tau c D_{k}}{2 T}\left\{\rho\left[T^{2}+2 T(N-M)\right]\right\} \\
& -\frac{\tau v D_{e} I_{e}}{2 T}\left\{(1-\rho)\left[T^{2}+2 T(M-T)\right]\right\}
\end{aligned}
$$




$$
\begin{aligned}
T C_{9}(T) & =\frac{o}{T}+\frac{h}{T}\left\{\frac{D}{2} t_{d}^{2}+D\left(1+m-t_{d}\right) \cdot t_{d} \cdot \ln \left(\frac{1+m-t_{d}}{1+m-T}\right)+\frac{p D^{2}}{x(1-p)^{2}}\left[t_{d}+\left(1+m-t_{d}\right)\right.\right. \\
& \left.\left.\times \ln \left(\frac{1+m-t_{d}}{1+m-T}\right)\right]^{2}+\frac{D}{2}\left(1+m-t_{d}\right)^{2} \cdot \ln \left(\frac{1+m-t_{d}}{1+m-T}\right)+\frac{D}{4}(1+m-T)^{2}-\frac{D}{4}\left(1+m-t_{d}\right)^{2}\right\} \\
& +\frac{(c+s) D}{(1-p) T}\left[t_{d}+\left(1+m-t_{d}\right) \cdot \ln \left(\frac{1+m-t_{d}}{1+m-T}\right)\right]+\frac{c D}{T}\left[t_{d}+\left(1+m-t_{d}\right) \cdot \ln \left(\frac{1+m-t_{d}}{1+m-T}\right)-T\right] \\
& +\frac{c I_{k} D T}{T}[\alpha(N+L)+\beta N]+\frac{c I_{k} D T^{2}}{2 T}(\alpha+\beta)+\frac{\tau c D I_{k}}{2 T}\left\{\rho\left[T^{2}+2 T(N-M)\right]\right. \\
& \left.+(1-\rho)(T-M)^{2}\right\}-\frac{\tau v D I_{e}}{2 T}(1-\rho) M^{2}
\end{aligned}
$$

Since $T C_{7}\left(t_{d}\right)=T C_{8}\left(t_{d}\right)$ and $T C_{8}(M)=T C_{9}(M), T C(T)$ are continuous and welldefined on $T>0$.

Case (II-2). Suppose that $t_{d} \geq M$

$$
T C(T)=\left\{\begin{array}{clc}
T C_{7}(T) & \text { if } & 0<T<M \\
T C_{10}(T) & \text { if } & M \leq T<t_{d} \\
T C_{9}(T) & \text { if } & t_{d} \leq T \leq R^{*}
\end{array}\right.
$$

where

$$
\begin{aligned}
T C_{10}(T) & =\frac{o}{T}+\frac{h D}{(1-p)}\left[\frac{(1-p) T}{2}+\frac{p D T}{x(1-p)}\right]+\frac{(c+s) D}{1-p}+\frac{c I_{k} D T}{T}[\alpha(N+L)+\beta N] \\
& +\frac{c I_{k} D T^{2}}{2 T}(\alpha+\beta)+\frac{\tau c D I_{k}}{2 T}\left\{\rho\left[T^{2}+2 T(N-M)\right]+(1-\rho)(T-M)^{2}\right\} \\
& -\frac{\tau v D I_{e}}{2 T}(1-\rho) M^{2}
\end{aligned}
$$

Since $T C_{7}(M)=T C_{10}(M)$ and $T C_{10}\left(t_{d}\right)=T C_{9}\left(t_{d}\right), T C(T)$ is continuous and welldefined on $T>0$.

\section{The Convexity and Monotonicity Properties of $T C_{i}(T)$} $(i=1,2,3,4,5,6,7,8,9,10)$

In this section, we continue the derivations described in above section and adopt Equations (13)-(16), (18), (20), (22)-(24) and (26) to find the first-order and the second-order derivatives of the annual total relevant costs $T C_{i}(T)$ with respect to $T$ in order to obtain the convexity properties as follows:

$$
\begin{aligned}
& T C^{\prime}{ }_{1}(T)= \frac{1}{T^{2}}\left\{-o+\frac{h D}{2}\left[1+\frac{2 p D}{x(1-p)^{2}}\right] T^{2}+\frac{c I_{k} D}{2}(\alpha+\beta) T^{2}+\frac{\tau v D I_{e}}{2} T^{2}\right\} \\
& T C^{\prime \prime}{ }_{1}(T)=\frac{2 o}{T^{3}}>0 \\
& T C^{\prime}{ }_{2}(T)= \frac{1}{T^{2}}\left\{-o+h \cdot G(T)+\left[\frac{(2-p) c+s}{1-p}\right] \cdot\left[D\left(1+m-t_{d}\right) \cdot \frac{T}{1+m-T}-D t_{d}\right.\right. \\
&-\left.\left.D\left(1+m-t_{d}\right) \cdot \ln \left(\frac{1+m-t_{d}}{1+m-T}\right)\right]+\frac{c I_{k} D}{2}(\alpha+\beta) T^{2}+\frac{\tau v D I_{e}}{2} T^{2}\right\} \\
& T C^{\prime \prime}{ }_{2}(T)= \frac{1}{T^{3}}\left\{2 o+h \cdot H(T)+\left[\frac{(2-p) c+s}{1-p}\right] \cdot\left[D\left(1+m-t_{d}\right) \cdot \frac{(1+m) T}{(1+m-T)^{2}}\right.\right. \\
&-\left.\left.3 D\left(1+m-t_{d}\right) \frac{T}{1+m-T}+2 D t_{d}+2 D\left(1+m-t_{d}\right) \cdot \ln \left(\frac{1+m-t_{d}}{1+m-T}\right)\right]\right\} \\
& T C^{\prime}{ }_{3}(T)= \frac{1}{T^{2}}\left\{-o+h \cdot G(T)+\left[\frac{(2-p) c+s}{1-p}\right] \cdot\left[D\left(1+m-t_{d}\right) \cdot \frac{T}{1+m-T}-D t_{d}\right.\right. \\
&-D\left(1+m-t_{d}\right) \cdot \ln \left(\frac{1+m-t_{d}}{1+m-T}\right)+\frac{c I_{k} D}{2}(\alpha+\beta) T^{2}+\frac{\tau c D I_{k}}{2}\left\{\rho\left[T^{2}-(N-M)^{2}\right]\right\} \\
&\left.+\frac{\tau v D I_{e}}{2}\left[\rho(M-N)^{2}+(1-\rho) T^{2}\right]\right\} \\
& \frac{1}{T^{3}}\left\{2 o+h \cdot H(T)+\left[\frac{(2-p) c+s}{1-p}\right] \cdot\left[D\left(1+m-t_{d}\right) \cdot \frac{(1+m) T}{(1+m-T)^{2}}\right.\right. \\
&\left.-3 D\left(1+m-t_{d}\right) \frac{T}{1+m-T}+2 D t_{d}+2 D\left(1+m-t_{d}\right) \cdot \ln \left(\frac{1+m-t_{d}}{1+m-T}\right)\right] \\
&\left.+\tau c D I_{k} \rho(N-M)^{2}-\tau v D I_{e} \rho(M-N)^{2}\right\} \\
& T C^{\prime \prime}{ }_{3}(T)
\end{aligned}
$$




$$
\begin{aligned}
& T C^{\prime}{ }_{4}(T)= \frac{1}{T^{2}}\left\{-o+h \cdot G(T)+\left[\frac{(2-p) c+s}{1-p}\right] \cdot\left[D\left(1+m-t_{d}\right) \cdot \frac{T}{1+m-T}-D t_{d}\right.\right. \\
&\left.-D\left(1+m-t_{d}\right) \cdot \ln \left(\frac{1+m-t_{d}}{1+m-T}\right)\right]+\frac{c I_{k} D}{2}(\alpha+\beta) T^{2}+\frac{\tau c D I_{k}}{2}\left\{\rho\left[T^{2}-(N-M)^{2}\right]\right. \\
&\left.\left.+(1-\rho)\left(T^{2}-M^{2}\right)\right\}+\frac{\tau v D I_{e}}{2}\left[\rho(M-N)^{2}+(1-\rho) M^{2}\right]\right\} \\
& T C^{\prime \prime}{ }_{4}(T)= \frac{1}{T^{3}}\left\{2 o+h \cdot H(T)+\left[\frac{(2-p) c+s}{1-p}\right] \cdot\left[D\left(1+m-t_{d}\right) \cdot \frac{(1+m) T}{(1+m-T)^{2}}\right.\right. \\
&\left.-3 D\left(1+m-t_{d}\right) \frac{T}{1+m-T}+2 D t_{d}+2 D\left(1+m-t_{d}\right) \cdot \ln \left(\frac{1+m-t_{d}}{1+m-T}\right)\right] \\
&\left.+\tau c D I_{k}\left[\rho(N-M)^{2}+(1-\rho) M^{2}\right]-\tau v D I_{e}\left[\rho(M-N)^{2}+(1-\rho) M^{2}\right]\right\} \\
& T C^{\prime}{ }_{5}(T)=\frac{1}{T^{2}}\left\{-o+\frac{h D}{2}\left[1+\frac{2 p D}{x(1-p)^{2}}\right] T^{2}+\frac{c I_{k} D}{2}(\alpha+\beta) T^{2}+\frac{\tau c D I_{k}}{2}\left\{\rho\left[T^{2}-(N-M)^{2}\right]\right\}\right.\left.\quad+\frac{\tau v D I_{e}}{2}\left[\rho(M-N)^{2}+(1-\rho) T^{2}\right]\right\} \\
& T C^{\prime \prime}{ }_{5}(T)=\frac{1}{T^{3}}\left\{2 o+\tau c D I_{k} \rho(N-M)^{2}-\tau v D I_{e} \rho(M-N)^{2}\right\} \\
& T C^{\prime}{ }_{6}(T)= \frac{1}{T^{2}}\left\{-o+\frac{h D}{2}\left[1+\frac{2 p D}{x(1-p)^{2}}\right] T^{2}+\frac{c I_{k} D}{2}(\alpha+\beta) T^{2}+\frac{\tau c D I_{k}}{2}\left\{\rho\left[T^{2}-(N-M)^{2}\right]\right.\right. \\
&\left.\left.+(1-\rho)\left(T^{2}-M^{2}\right)\right\}+\frac{\tau v D I_{e}}{2}\left[\rho(M-N)^{2}+(1-\rho) M^{2}\right]\right\}
\end{aligned}
$$

$$
\begin{aligned}
T C^{\prime}{ }_{8}(T) & =\frac{1}{T^{2}}\left\{-o+h \cdot G(T)+\left[\frac{(2-p) c+s}{1-p}\right]\left[D\left(1+m-t_{d}\right) \frac{T}{1+m-T}\right.\right. \\
& \left.-D t_{d}-D\left(1+m-t_{d}\right) \cdot \ln \left(\frac{1+m-t_{d}}{1+m-T}\right)\right]+\frac{c I_{k} D}{2}(\alpha+\beta) T^{2}+\frac{\tau c D I_{k}}{2} \rho T^{2} \\
& \left.+\frac{\tau v D I_{e}}{2}(1-\rho) T^{2}\right\}
\end{aligned}
$$

$$
\begin{aligned}
T C^{\prime \prime}{ }_{8}(T) & =\frac{1}{T^{3}}\left\{2 o+h \cdot H(T)+\left[\frac{(2-p) c+s}{1-p}\right]\left[D\left(1+m-t_{d}\right) \frac{(1+m) T}{(1+m-T)^{2}}\right.\right. \\
& \left.\left.-3 D\left(1+m-t_{d}\right) \frac{T}{1+m-T}+2 D t_{d}+2 D\left(1+m-t_{d}\right) \cdot \ln \left(\frac{1+m-t_{d}}{1+m-T}\right)\right]\right\}
\end{aligned}
$$

$$
\begin{aligned}
T C^{\prime}{ }_{9}(T) & =\frac{1}{T^{2}}\left\{-o+h \cdot G(T)+\left[\frac{(2-p) c+s}{1-p}\right]\left[D\left(1+m-t_{d}\right) \frac{T}{1+m-T}\right.\right. \\
& \left.-D t_{d}-D\left(1+m-t_{d}\right) \cdot \ln \left(\frac{1+m-t_{d}}{1+m-T}\right)\right]+\frac{c I_{k} D}{2}(\alpha+\beta) T^{2}+\frac{\tau c D I_{k}}{2}\left[T^{2}-(1-\rho) M^{2}\right] \\
& \left.+\frac{\tau v D I_{e}}{2}(1-\rho) M^{2}\right\}
\end{aligned}
$$

$$
\begin{aligned}
T C^{\prime \prime}{ }_{9}(T) & =\frac{1}{T^{3}}\left\{2 o+h \cdot H(T)+\left[\frac{(2-p) c+s}{1-p}\right]\left[D\left(1+m-t_{d}\right) \frac{(1+m) T}{(1+m-T)^{2}}\right.\right. \\
& \left.-3 D\left(1+m-t_{d}\right) \frac{T}{1+m-T}+2 D t_{d}+2 D\left(1+m-t_{d}\right) \cdot \ln \left(\frac{1+m-t_{d}}{1+m-T}\right)\right] \\
& \left.+\tau c D I_{k}(1-\rho) M^{2}-\tau v D I_{e}(1-\rho) M^{2}\right\}
\end{aligned}
$$

$$
\begin{aligned}
T C^{\prime}{ }_{10}(T) & =\frac{1}{T^{2}}\left\{-o+\frac{h D}{2}\left[1+\frac{2 p D}{x(1-p)^{2}}\right] T^{2}+\frac{c I_{k} D}{2}(\alpha+\beta) T^{2}+\frac{\tau c D I_{k}}{2}\left[T^{2}-(1-\rho) M^{2}\right]\right. \\
& \left.+\frac{\tau v D I_{e}}{2}(1-\rho) M^{2}\right\}
\end{aligned}
$$

and

$$
T C^{\prime \prime}{ }_{10}(T)=\frac{1}{T^{3}}\left\{2 o+\tau c D I_{k}(1-\rho) M^{2}-\tau v D I_{e}(1-\rho) M^{2}\right\}
$$

where

$$
\begin{aligned}
G(T) & =D\left(1+m-t_{d}\right) \cdot t_{d} \frac{T}{1+m-T}+\frac{2 p D^{2}}{x(1-p)^{2}}\left(1+m-t_{d}\right)\left[t_{d}+\left(1+m-t_{d}\right)\right. \\
& \left.\times \ln \left(\frac{1+m-t_{d}}{1+m-T}\right)\right] \cdot \frac{T}{1+m-T}+\frac{D}{2}\left(1+m-t_{d}\right)^{2} \cdot \frac{T}{1+m-T}-\frac{D}{2}(1+m-T) T \\
& -\frac{D}{2} t_{d}^{2}-D\left(1+m-t_{d}\right) \cdot t_{d} \cdot \ln \left(\frac{1+m-t_{d}}{1+m-T}\right)-\frac{p D^{2}}{x(1-p)^{2}}\left[t_{d}+\left(1+m-t_{d}\right) \cdot \ln \left(\frac{1+m-t_{d}}{1+m-T}\right)\right]^{2} \\
& -\frac{D}{2}\left(1+m-t_{d}\right)^{2} \cdot \ln \left(\frac{1+m-t_{d}}{1+m-T}\right)-\frac{D}{4}(1+m-T)^{2}+\frac{D}{4}\left(1+m-t_{d}\right)^{2}
\end{aligned}
$$


and

$$
\begin{aligned}
H(T) & =D\left(1+m-t_{d}\right) t_{d} \frac{T^{2}}{(1+m-T)^{2}}+\frac{D}{2}\left(1+m-t_{d}\right)^{2} \frac{T^{2}}{(1+m-T)^{2}}+\frac{D}{2} T^{2} \\
& -2 D\left(1+m-t_{d}\right) \cdot t_{d} \frac{T}{1+m-T}-D\left(1+m-t_{d}\right)^{2} \frac{T}{1+m-T}+D(1+m-T) T \\
& +D t_{d}^{2}+2 D\left(1+m-t_{d}\right) t_{d} \cdot \ln \left(\frac{1+m-t_{d}}{1+m-T}\right)+D\left(1+m-t_{d}\right)^{2} \ln \left(\frac{1+m-t_{d}}{1+m-T}\right) \\
& +\frac{D}{2}(1+m-T)^{2}-\frac{D}{2}\left(1+m-t_{d}\right)^{2}+\frac{2 p D^{2}}{x(1-p)^{2}}\left(1+m-t_{d}\right)^{2} \frac{T^{2}}{(1+m-T)^{2}} \\
& +\frac{2 p D^{2}}{x(1-p)^{2}}\left(1+m-t_{d}\right)\left[t_{d}+\left(1+m-t_{d}\right) \cdot \ln \left(\frac{1+m-t_{d}}{1+m-T}\right)\right] \frac{T^{2}}{(1+m-T)^{2}} \\
& -\frac{4 p D^{2}}{x(1-p)^{2}}\left(1+m-t_{d}\right)\left[t_{d}+\left(1+m-t_{d}\right) \cdot \ln \left(\frac{1+m-t_{d}}{1+m-T}\right)\right] \frac{T}{1+m-T} \\
& +\frac{2 p D^{2}}{x(1-p)^{2}}\left[t_{d}+\left(1+m-t_{d}\right) \cdot \ln \left(\frac{1+m-t_{d}}{1+m-T}\right)\right]^{2}
\end{aligned}
$$

Obviously, it is shown that $T C_{1}(T)$ and $T C_{7}(T)$ are convex functions on $(0, \infty)$, respectively.

Now, we let

$$
\begin{gathered}
W_{1}=2 o-\tau v I_{e} D \rho(M-N)^{2} \\
W_{2}=2 o-\tau v I_{e} D\left[\rho(M-N)^{2}+(1-\rho) M^{2}\right]
\end{gathered}
$$

and

$$
W_{3}=2 o-\tau v I_{e} D(1-\rho) M^{2}
$$

Then, we have the following convexity results.

Lemma 1. Each of the following assertions holds true:

(A) $T C_{r}(T)(r=2,8)$ is convex on $\left[t_{d}, \infty\right)$.

(B) If $W_{2} \geq 0$, then $T C_{l}(T)(l=3,4,5,6,9,10)$ is convex on $\left[t_{d}, \infty\right)$.

(C) If $W_{1}<0$, then $T C_{i}(T)(i=3,5)$ is increasing on $\left[t_{d}, \infty\right)$.

(D) If $W_{2}<0$, then $T C_{j}(T)(j=4,6)$ is increasing on $\left[t_{d}, \infty\right)$.

(E) If $W_{3}<0$, then $T C_{k}(T)(k=9,10)$ is increasing on $\left[t_{d}, \infty\right)$.

Remark 1. In our proof of Lemma 1, we need the assertions of Lemma 2.

Lemma 2. Each of the following assertions holds true:
(A) $G(T)>0$ if $T \geq t_{d}$
(B) $D\left(1+m-t_{d}\right) \frac{T}{1+m-T}-D t_{d}-D\left(1+m-t_{d}\right) \cdot \ln \left(\frac{1+m-t_{d}}{1+m-T}\right)>0$ if $T \geq t_{d}$
(C) $H(T)>0$ if $T \geq t_{d}$
$D\left(1+m-t_{d}\right) \frac{(1+m) T}{(1+m-T)^{2}}-3 D\left(1+m-t_{d}\right) \frac{T}{1+m-T} \quad$ if $T \geq t_{d}$
$+2 D t_{d}+2 D\left(1+m-t_{d}\right) \cdot \ln \left(\frac{1+m-t_{d}}{1+m-T}\right)>0$

Remark 2. The proof of Lemma 2 is given in the Appendix A.

\section{Proof of Lemma 1.}

(A) Lemma 2(C) and 2(D) reveal that $T C^{\prime \prime}{ }_{r}(T)>0(r=2,8)$ for $T \geq t_{d}$. Furthermore, $T C_{r}(T)(r=2,8)$ is convex on $\left[t_{d}, \infty\right)$.

(B) If $W_{2}>0$, then $W_{1}>0$ and $W_{3}>0$. Moreover, Equations (32), (34), (36), (38), (44) and (46), together with Lemma 2(C) and 2(D), yield

$$
T C^{\prime \prime}{ }_{i}(T)>\frac{1}{T^{3}}\left\{2 o-\tau v D I_{e} \rho(M-N)^{2}\right\}>0, \quad i=3 \text { and } 5
$$




$$
T C^{\prime \prime}{ }_{j}(T)>\frac{1}{T^{3}}\left\{2 o-\tau v D I_{e}\left[\rho(M-N)+(1-\rho) M^{2}\right]\right\}>0, j=4 \text { and } 6
$$

and

$$
T C^{\prime \prime}{ }_{k}(T)>\frac{1}{T^{3}}\left\{2 o-\tau v D I_{\mathcal{e}}\left[(1-\rho) M^{2}\right]\right\}>0, \quad k=9 \text { and } 10
$$

The above results imply that $T C_{l}(T) \quad(l=3,4,5,6,9,10)$ is convex on $\left[t_{d}, \infty\right)$.

(C) When $W_{1}<0$, from Equations (31) and (33) and Lemma 2(A) and 2(B), we have the following results:

$T^{\prime}{ }_{i}(T)>\frac{1}{2 T^{2}}\left\{-2 o+\tau v D I_{e} \rho(M-N)^{2}\right\}>0, \quad i=3$ and 5

Furthermore, $T C_{i}(T)(i=3,5)$ is increasing on $\left[T_{d}, \infty\right)$.

(D) When $W_{2}<0$, from Equations (35) and (37) and Lemma 2(A) and 2(B), we have the following results:

$$
T C^{\prime}{ }_{j}(T)>\frac{1}{2 T^{2}}\left\{-2 o+\tau v D I_{e}\left[\rho(M-N)^{2}+(1-\rho) M^{2}\right\}>0, \quad j=4 \quad \text { and } 6\right.
$$

Furthermore, $T C_{j}(T)(j=4,6)$ is increasing on $\left[t_{d}, \infty\right)$.

(E) When $W_{3}<0$, from Equations (43) and (45) and Lemma 2(A) and 2(B), we have the following results:

$$
T C^{\prime}{ }_{k}(T)>\frac{1}{2 T^{2}}\left\{-2 o+\tau v D I_{\mathcal{e}}(1-\rho) M^{2}\right\}>0, \quad k=9 \text { and } 10
$$

Furthermore, $T C_{k}(T)(k=9,10)$ is increasing on $\left[t_{d}, \infty\right)$. This completes the proof of Lemma 1.

\section{The Main Theorems for Optimal Replenishment Cycle Time $T^{*}$ of $T C(T)$}

In this section, we apply the convexity and monotonicity properties in order to develop efficient decision rules for the optimal replenishment cycle time $T^{*}$ of $T C(T)$.

4.1. Decision Rule of the Optimal Replenishment Cycle Time $T^{*}$ When $N \leq M$

4.1.1. Decision Rule of the Optimal Replenishment Cycle Time $T^{*}$ When $t_{d}<M-N<M$

From Equation (12), we have

$$
T C(T)=\left\{\begin{array}{ccc}
T C_{1}(T) & \text { if } & 0<T<t_{d} \\
T C_{2}(T) & \text { if } & t_{d} \leq T<M-N \\
T C_{3}(T) & \text { if } & M-N \leq T<M \\
T C_{4}(T) & \text { if } & M \leq T<R^{*}
\end{array}\right.
$$

All $T C_{i}(T)(i=1,2,3,4)$ and $T C(T)$ are defined on $T>0$. From Equations (27), (29), (31) and (33), we have

$$
\begin{gathered}
T C^{\prime}{ }_{1}\left(t_{d}\right)=T C^{\prime}{ }_{2}\left(t_{d}\right)=\frac{\Delta_{1}}{t_{d}^{2}} \\
T C^{\prime}{ }_{2}(M-N)=T C^{\prime}{ }_{3}(M-N)=\frac{\Delta_{2}}{(M-N)^{2}} \\
T C^{\prime}{ }_{3}(M)=T C^{\prime}{ }_{4}(M)=\frac{\Delta_{3}}{M^{2}}
\end{gathered}
$$

and

where

$$
\mathrm{TC}^{\prime}{ }_{4}\left(R^{*}\right)=\frac{\Delta^{*}}{R^{* 2}}
$$

$$
\Delta_{1}=-o+\frac{h D}{2}\left[1+\frac{2 p D}{x(1-p)^{2}}\right] t_{d}^{2}+\frac{c I_{k} D}{2}(\alpha+\beta) t_{d}^{2}+\frac{\tau v D I_{e}}{2} t_{d}^{2}
$$




$$
\begin{aligned}
\Delta_{2} & =-o+h \cdot G(M-N)+\left[\frac{(2-p) c+s}{1-p}\right] \cdot\left[D\left(1+m-t_{d}\right) \cdot \frac{(M-N)}{1+m-(M-N)}-D t_{d}\right. \\
& \left.-D\left(1+m-t_{d}\right) \cdot \ln \left(\frac{1+m-t_{d}}{1+m-(M-N)}\right)\right]+\frac{c I_{k} D}{2}(\alpha+\beta)(M-N)^{2}+\frac{\tau v D I_{e}}{2}(M-N)^{2} \\
\Delta_{3} & =-o+h \cdot G(M)+\left[\frac{(2-p) c+s}{1-p}\right] \cdot\left[D\left(1+m-t_{d}\right) \cdot \frac{M}{1+m-M}-D t_{d}\right. \\
& \left.-D\left(1+m-t_{d}\right) \cdot \ln \left(\frac{1+m-t_{d}}{1+m-M}\right)\right]+\frac{c I_{k} D}{2}(\alpha+\beta) M^{2}+\frac{\tau c D I_{k}}{2}\left\{\rho\left[M^{2}-(N-M)^{2}\right]\right\} \\
& +\frac{\tau v D I_{e}}{2}\left[\rho(M-N)^{2}+(1-\rho) M^{2}\right]
\end{aligned}
$$

and

$$
\begin{aligned}
\Delta^{*} & =-o+h \cdot G\left(R^{*}\right)+\left[\frac{(2-p) c+s}{1-p}\right] \cdot\left[D\left(1+m-t_{d}\right) \cdot \frac{R^{*}}{1+m-R^{*}}-D t_{d}\right. \\
& \left.-D\left(1+m-t_{d}\right) \cdot \ln \left(\frac{1+m-t_{d}}{1+m-R^{*}}\right)\right]+\frac{c I_{k} D}{2}(\alpha+\beta) R^{* 2}+\frac{\tau c D I_{k}}{2}\left\{\rho\left[R^{* 2}-(N-M)^{2}\right]\right. \\
& \left.+(1-\rho)\left(R^{* 2}-M^{2}\right)\right\}+\frac{\tau v D I_{e}}{2}\left[\rho(M-N)^{2}+(1-\rho) M^{2}\right]
\end{aligned}
$$

From the above results, we have $\Delta_{1}<\Delta_{2}$. In addition, if $W_{1} \geq 0$, then $\Delta_{1}<\Delta_{2}<$ $\Delta_{3}<\Delta^{*}$. Otherwise, if $W_{1}<0$, we obtain $0<\Delta_{2}<\Delta_{3}<\Delta^{*}$. From the above discussions, the following results are achieved.

Theorem 1. Suppose that $t_{d}<M-N<M$. Then, each of the following results holds true:

(I) If $W_{1} \geq 0$, then
(A) If $\Delta_{1}<0, \Delta_{2}<0, \Delta_{3}<0$ and $\Delta^{*}<0$, then TC $\left(T^{*}\right)=T C_{4}\left(R^{*}\right)$.
(B) If $\Delta_{1}<0, \Delta_{2}<0, \Delta_{3}<0$ and $\Delta^{*} \geq 0$, then $\mathrm{TC}\left(T^{*}\right)=\mathrm{TC}_{4}\left(T_{4}^{*}\right)$.
(C) If $\Delta_{1}<0, \Delta_{2}<0, \Delta_{3} \geq 0$ and $\Delta^{*} \geq 0$, then TC $\left(T^{*}\right)=T C_{3}\left(T_{3}^{*}\right)$.
(D) If $\Delta_{1}<0, \Delta_{2} \geq 0, \Delta_{3} \geq 0$ and $\Delta^{*} \geq 0$, then $T C\left(T^{*}\right)=T C_{2}\left(T_{2}^{*}\right)$.
(E) If $\Delta_{1} \geq 0, \Delta_{2} \geq 0, \Delta_{3} \geq 0$ and $\Delta^{*} \geq 0$, then $T C\left(T^{*}\right)=T C_{1}\left(T_{1}^{*}\right)$.

(II) If $W_{1}<0$, then

(A) If $\Delta_{1}<0, \Delta_{2} \geq 0, \Delta_{3} \geq 0$ and $\Delta^{*} \geq 0$, then $T C\left(T^{*}\right)=T C_{2}\left(T_{2}^{*}\right)$.

(B) If $\Delta_{1} \geq 0, \Delta_{2} \geq 0, \Delta_{3} \geq 0$ and $\Delta^{*} \geq 0$, then $\operatorname{TC}\left(T^{*}\right)=T C_{1}\left(T_{1}^{*}\right)$.

Proof. The proof of Theorem 1 follows immediately from the above discussions.

4.1.2. Decision Rule of the Optimal Replenishment Cycle Time $T^{*}$ When $M-N<t_{d}<M$ From Equation (17), we have

$$
T C(T)=\left\{\begin{array}{clc}
T C_{1}(T) & \text { if } & 0<T<M-N \\
T C_{5}(T) & \text { if } & M-N \leq T<t_{d} \\
T C_{3}(T) & \text { if } & t_{d} \leq T<M \\
T C_{4}(T) & \text { if } & M \leq T \leq R^{*}
\end{array}\right.
$$

Herein, $T C_{5}(T)$ is defined on $T>0$ as well. Equations (27), (31) and (35) imply that

$$
T C^{\prime}{ }_{1}(M-N)=T C^{\prime}{ }_{5}(M-N)=\frac{\Delta_{4}}{(M-N)^{2}}
$$

and

$$
T C^{\prime}{ }_{5}\left(t_{d}\right)=T C_{3}^{\prime}\left(t_{d}\right)=\frac{\Delta_{5}}{t_{d}^{2}}
$$

where

$$
\Delta_{4}=-o+\frac{h D}{2}\left[1+\frac{2 p D}{x(1-p)^{2}}\right](M-N)^{2}+\frac{c I_{k} D}{2}(\alpha+\beta)(M-N)^{2}+\frac{\tau v D I_{e}}{2}(M-N)^{2}
$$

and

$$
\begin{aligned}
& \Delta_{5}=-o+\frac{h D}{2}\left[1+\frac{2 p D}{x(1-p)^{2}}\right] t_{d}^{2}+\frac{c I_{k} D}{2}(\alpha+\beta) t_{d}^{2}+\frac{\tau c D I_{k}}{2}\left\{\rho\left[t_{d}^{2}-(N-M)^{2}\right]\right\} \\
& \left.+\frac{\tau v D I_{e}}{2}\left[\rho(M-N)^{2}+(1-\rho) t_{d}^{2}\right]\right\}
\end{aligned}
$$


From the above results, we have these three situations: one is $\Delta_{4}<\Delta_{5}<\Delta_{3}<\Delta^{*}$ if $W_{2} \geq 0$, another is $\Delta_{4}<\Delta_{5}<\Delta_{3}<\Delta^{*}$ and $0<\Delta_{3}<\Delta^{*}$ if $W_{2}<0$ and $W_{1} \geq 0$, and the other is $0<\Delta_{4}<\Delta_{5}<\Delta_{3}<\Delta^{*}$ if $W_{1}<0$. Furthermore, we have the following results.

Theorem 2. Suppose that $M-N<t_{d}<M$ Then, each of the following results holds true:

(I) If $W_{2} \geq 0$, then
(A) If $\Delta_{4}<0, \Delta_{5}<0, \Delta_{3}<0$ and $\Delta^{*}<0$, then TC $\left(T^{*}\right)=T C_{4}\left(R^{*}\right)$.
(B) If $\Delta_{4}<0, \Delta_{5}<0, \Delta_{3}<0$ and $\Delta^{*} \geq 0$, then TC $\left(T^{*}\right)=T C_{4}\left(T_{4}^{*}\right)$.
(C) If $\Delta_{4}<0, \Delta_{5}<0, \Delta_{3} \geq 0$ and $\Delta^{*} \geq 0$, then TC $\left(T^{*}\right)=T C_{3}\left(T_{3}^{*}\right)$.
(D) If $\Delta_{4}<0, \Delta_{5} \geq 0, \Delta_{3} \geq 0$ and $\Delta^{*} \geq 0$, then TC $\left(T^{*}\right)=T C_{5}\left(T_{5}^{*}\right)$.
(E) If $\Delta_{4} \geq 0, \Delta_{5} \geq 0, \Delta_{3} \geq 0$ and $\Delta^{*} \geq 0$, then TC $\left(T^{*}\right)=T C_{1}\left(T_{1}^{*}\right)$.

(II) If $W_{2}<0$ and $W_{1} \geq 0$, then

(A) If $\Delta_{4}<0, \Delta_{5}<0$ and $\Delta_{3} \geq 0$, then $T C\left(T^{*}\right)=T C_{3}\left(T_{3}^{*}\right)$.

(B) If $\Delta_{4}<0, \Delta_{5} \geq 0$ and $\Delta_{3} \geq 0$, then $T C\left(T^{*}\right)=T C_{5}\left(T_{5}^{*}\right)$.

(C) If $\Delta_{4} \geq 0, \Delta_{5} \geq 0$ and $\Delta_{3} \geq 0$, then $T C\left(T^{*}\right)=T C_{1}\left(T_{1}^{*}\right)$.

(III) If $W_{1}<0$, then

(A) If $\Delta_{4} \geq 0, \Delta_{5} \geq 0$ and $\Delta_{3} \geq 0$, then $T C\left(T^{*}\right)=T C_{1}\left(T_{1}^{*}\right)$.

Proof. The proof of Theorem 2 follows immediately from the above discussions.

4.1.3. The Decision Rule of the Optimal Replenishment Cycle Time $T^{*}$ When $M-N<M<t_{d}$

$$
T C(T)=\left\{\begin{array}{ccc}
T C_{1}(T) & \text { if } & 0<T<M-N \\
T C_{5}(T) & \text { if } & M-N \leq T<M \\
T C_{6}(T) & \text { if } & M \leq T<t_{d} \\
T C_{4}(T) & \text { if } & t_{d} \leq T \leq R^{*}
\end{array}\right.
$$

Likewise, $T C_{6}(T)$ is defined on $T>0$, Equations (33), (35) and (37) imply that

$$
T C^{\prime}{ }_{5}(M)=T C^{\prime}{ }_{6}(M)=\frac{\Delta_{6}}{M^{2}}
$$

and

$$
T C^{\prime}{ }_{6}\left(t_{d}\right)=T C_{4}^{\prime}\left(t_{d}\right)=\frac{\Delta_{7}}{t_{d}^{2}}
$$

where

$$
\begin{aligned}
\Delta_{6} & =-o+\frac{h D}{2}\left[1+\frac{2 p D}{x(1-p)^{2}}\right] M^{2}+\frac{c I_{k} D}{2}(\alpha+\beta) M^{2}+\frac{\tau c D I_{k}}{2}\left\{\rho\left[M^{2}-(N-M)^{2}\right]\right\} \\
& +\frac{\tau v D I_{e}}{2}\left[\rho(M-N)^{2}+(1-\rho) M^{2}\right]
\end{aligned}
$$

and

$$
\begin{aligned}
\Delta_{7} & =-o+\frac{h D}{2}\left[1+\frac{2 p D}{x(1-p)^{2}}\right] t_{d}^{2}+\frac{c I_{k} D}{2}(\alpha+\beta) t_{d}^{2}+\frac{\tau c D I_{k}}{2}\left\{\rho\left[t_{d}^{2}-(N-M)^{2}\right]\right. \\
& \left.+(1-\rho)\left(t_{d}^{2}-M^{2}\right)\right\}+\frac{\tau v D I_{e}}{2}\left[\rho(M-N)^{2}+(1-\rho) M^{2}\right]
\end{aligned}
$$

In addition, there are three situations to occur here: one is $\Delta_{4}<\Delta_{6}<\Delta_{7}<\Delta^{*}$ when $W_{2} \geq 0$, another is $\Delta_{4}<\Delta_{6}$ and $0<\Delta_{6}<\Delta_{7}<\Delta^{*}$ when $W_{2}<0$ and $W_{1} \geq 0$, and the other is $0<\Delta_{4}<\Delta_{6}<\Delta_{7}<\Delta^{*}$ when $W_{1}<0$. Furthermore, we have the following results.

Theorem 3. Suppose that $M-N<M<t_{d}$. Then, each of the the following results holds true: (I) If $W_{2} \geq 0$, then

$$
\text { (A) If } \Delta_{4}<0, \Delta_{6}<0, \Delta_{7}<0 \text { and } \Delta^{*}<0 \text {, then } \operatorname{TC}\left(T^{*}\right)=T C_{4}\left(R^{*}\right) \text {. }
$$


(B) If $\Delta_{4}<0, \Delta_{6}<0, \Delta_{7}<0$ and $\Delta^{*} \geq 0$, then $T C\left(T^{*}\right)=T C_{4}\left(T_{4}^{*}\right)$.

(C) If $\Delta_{4}<0, \Delta_{6}<0, \Delta_{7} \geq 0$ and $\Delta^{*} \geq 0$, then TC $\left(T^{*}\right)=T C_{6}\left(T_{6}^{*}\right)$.

(D) If $\Delta_{4}<0, \Delta_{6} \geq 0, \Delta_{7} \geq 0$ and $\Delta^{*} \geq 0$, then $T C\left(T^{*}\right)=T C_{5}\left(T_{5}^{*}\right)$.

(E) If $\Delta_{4} \geq 0, \Delta_{6} \geq 0, \Delta_{7} \geq 0$ and $\Delta^{*} \geq 0$, then $T C\left(T^{*}\right)=T C_{1}\left(T_{1}^{*}\right)$.

(II) If $W_{2}<0$ and $W_{1} \geq 0$, then

(A) If $\Delta_{4}<0, \Delta_{6} \geq 0, \Delta_{7} \geq 0$ and $\Delta^{*} \geq 0$, then $T C\left(T^{*}\right)=T C_{5}\left(T_{5}^{*}\right)$.

(B) If $\Delta_{4} \geq 0, \Delta_{6} \geq 0, \Delta_{7} \geq 0$ and $\Delta^{*} \geq 0$, then $T C\left(T^{*}\right)=T C_{1}\left(T_{1}^{*}\right)$.

(III) If $W_{1}<0$, then $\Delta_{4}>0$ and $T C\left(T^{*}\right)=T C_{1}\left(T_{1}^{*}\right)$.

Proof. The proof of Theorem 3 follows immediately from the above discussions.

4.2. The Decision Rule of the Optimal Replenishment Cycle Time $T^{*}$ When $N>M$

4.2.1. The Decision Rule of the Optimal Replenishment Cycle Time $T^{*}$ When $t_{d}<M$

From Equation (21), we have

$$
T C(T)=\left\{\begin{array}{ccc}
T C_{7}(T) & \text { if } & 0<T<t_{d} \\
T C_{8}(T) & \text { if } & t_{d} \leq T<M \\
T C_{9}(T) & \text { if } & M \leq T \leq R^{*}
\end{array}\right.
$$

All $T C_{v}(T)(v=7,8,9)$ are defined on $T>0$. From Equations (39), (41), and (43), we have

$$
\begin{gathered}
T C^{\prime}{ }_{7}\left(t_{d}\right)=T C^{\prime}{ }_{8}\left(t_{d}\right)=\frac{\Delta_{8}}{t_{d}^{2}} \\
T C^{\prime}{ }_{8}(M)=T C^{\prime}{ }_{9}(M)=\frac{\Delta_{9}}{M^{2}}
\end{gathered}
$$

and

$$
T C^{\prime}{ }_{9}\left(R^{*}\right)=\frac{\Delta^{* *}}{R^{* 2}}
$$

where

$$
\begin{aligned}
\Delta_{8}=-o+\frac{h D}{2} & {\left[1+\frac{2 p D}{x(1-p)^{2}}\right] t_{d}^{2}+\frac{c I_{k} D}{2}(\alpha+\beta) t_{d}^{2}+\frac{\tau c D I_{k}}{2} \rho t_{d}^{2}+\frac{\tau v D I_{e}}{2}(1-\rho) t_{d}^{2} } \\
\Delta_{9} & =-o+h \cdot G(M)+\left[\frac{(2-p) c+s}{1-p}\right]\left[D\left(1+m-t_{d}\right) \frac{M}{1+m-M}-D t_{d}\right. \\
& \left.-D\left(1+m-t_{d}\right) \cdot \ln \left(\frac{1+m-t_{d}}{1+m-M}\right)\right]+\frac{c I_{k} D}{2}(\alpha+\beta) M^{2}+\frac{\tau c D I_{k}}{2} \rho M^{2} \\
& +\frac{\tau v D I_{e}}{2}(1-\rho) M^{2}
\end{aligned}
$$

and

$$
\begin{aligned}
\Delta^{* *} & =-o+h \cdot G\left(R^{*}\right)+\left[\frac{(2-p) c+s}{1-p}\right]\left[D\left(1+m-t_{d}\right) \frac{R^{*}}{1+m-R^{*}}-D t_{d}\right. \\
& \left.-D\left(1+m-t_{d}\right) \cdot \ln \left(\frac{1+m t_{d}}{1+m-R^{*}}\right)\right]+\frac{c I_{k} D}{2}(\alpha+\beta) R^{* 2}+\frac{\tau D I_{k}}{2}\left[R^{* 2}-(1-\rho) M^{2}\right] \\
& \left.+\frac{\tau v D I_{e}}{2}(1-\rho) M^{2}\right\}
\end{aligned}
$$

Additionally, if $W_{3} \geq 0$, then $\Delta_{8}<\Delta_{9}<\Delta^{* *}$. Otherwise, if $W_{3}<0$, we have $\Delta_{8}<\Delta_{9}$ and $0<\Delta_{9}<\Delta^{* *}$. Furthermore, we have the following Theorem.

Theorem 4. Suppose that $t_{d}<M$ Then, each of the following results holds true:

(I) If $W_{3} \geq 0$, then
(A) If $\Delta_{8}<0, \Delta_{9}<0$ and $\Delta^{* *}<0$, then $T C\left(T^{*}\right)=T C_{9}\left(R^{*}\right)$.
(B) If $\Delta_{8}<0, \Delta_{9}<0$ and $\Delta^{* *} \geq 0$, then TC $\left(T^{*}\right)=T C_{9}\left(T_{9}^{*}\right)$.
(C) If $\Delta_{8}<0, \Delta_{9} \geq 0$ and $\Delta^{* *} \geq 0$, then $T C\left(T^{*}\right)=T C_{8}\left(T_{8}^{*}\right)$.
(D) If $\Delta_{8} \geq 0, \Delta_{9} \geq 0$ and $\Delta^{* *} \geq 0$, then $T C\left(T^{*}\right)=T C_{7}\left(T_{7}^{*}\right)$.

(II) If $W_{3}<0$, then

(A) If $\Delta_{8}<0, \Delta_{9} \geq 0$ and $\Delta^{* *} \geq 0$, then $T C\left(T^{*}\right)=T C_{8}\left(T_{8}^{*}\right)$.

(B) If $\Delta_{8} \geq 0, \Delta_{9} \geq 0$ and $\Delta^{* *} \geq 0$, then $T C\left(T^{*}\right)=T C_{7}\left(T_{7}^{*}\right)$. 
Proof. The proof of Theorem 4 follows immediately from the above discussions.

4.2.2. Decision Rule of the Optimal Replenishment Cycle Time $T^{*}$ When $t_{d} \geq M$

From Equations (25), we have

$$
T C(T)=\left\{\begin{array}{clc}
T C_{7}(T) & \text { if } & 0<T<M \\
T C_{10}(T) & \text { if } & M \leq T<t_{d} \\
T C_{9}(T) & \text { if } & t_{d} \leq T \leq R^{*}
\end{array}\right.
$$

Herein, $T C_{10}(T)$ is defined on $T>0$. From Equations (39), (43), and (45), we have

$$
T C^{\prime}{ }_{7}(M)=T C^{\prime}{ }_{10}(M)=\frac{\Delta_{10}}{M^{2}}
$$

and

$$
T C^{\prime}{ }_{10}\left(t_{d}\right)=T C^{\prime}{ }_{9}\left(t_{d}\right)=\frac{\Delta_{11}}{t_{d}^{2}}
$$

where

$$
\left.\Delta_{10}=-o+\frac{h D}{2}\left[1+\frac{2 p D}{x(1-p)^{2}}\right] M^{2}+\frac{c I_{k} D}{2}(\alpha+\beta) M^{2}+\frac{\tau c D I_{k}}{2} \rho M^{2}+\frac{\tau v D I_{e}}{2}(1-\rho) M^{2}\right\}
$$

and

$$
\begin{aligned}
\Delta_{11} & =-o+\frac{h D}{2}\left[1+\frac{2 p D}{x(1-p)^{2}}\right] t_{d}^{2}+\frac{c I_{k} D}{2}(\alpha+\beta) t_{d}^{2}+\frac{\tau c D I_{k}}{2}\left[t_{d}^{2}-(1-\rho) M^{2}\right] \\
& \left.+\frac{\tau v D I_{e}}{2}(1-\rho) M^{2}\right\}
\end{aligned}
$$

Likewise, if $W_{3} \geq 0$, then $\Delta_{10}<\Delta_{11}<\Delta^{* *}$. Otherwise, if $W_{3}<0$, we have $0<\Delta_{10}<$ $\Delta_{11}<\Delta^{* *}$. From above arguments, we have the following theorem.

Theorem 5. Suppose that $t_{d} \geq M$ Then, each of the following results holds true:

(I) If $W_{3} \geq 0$, then
(A) If $\Delta_{10}<0, \Delta_{11}<0$ and $\Delta^{* *}<0$, then TC $\left(T^{*}\right)=T C_{9}\left(R^{*}\right)$.
(B) If $\Delta_{10}<0, \Delta_{11}<0$ and $\Delta^{* *} \geq 0$, then TC $\left(T^{*}\right)=T C_{9}\left(T_{9}^{*}\right)$.
(C) If $\Delta_{10}<0, \Delta_{11} \geq 0$ and $\Delta^{* *} \geq 0$, then $T C\left(T^{*}\right)=T C_{10}\left(T_{10}^{*}\right)$.
(D) If $\Delta_{10} \geq 0, \Delta_{11} \geq 0$ and $\Delta^{* *} \geq 0$, then $T C\left(T^{*}\right)=T C_{7}\left(T_{7}^{*}\right)$.

(II) If $W_{3}<0$, then $\Delta_{10} \geq 0$ and $T C\left(T^{*}\right)=T C_{7}\left(T_{7}^{*}\right)$.

Proof: The proof follows immediately from the above discussions.

\section{Illustrative Numerical Examples}

In this section, we will provide numerical examples to illustrate the theoretical results.

We assume that the maximum lifetime of the deteriorating items is 2 years $(m=2)$.

The computed results are shown in Tables 1-9. 
Table 1. The optimal replenishment policy used Theorem 1(I).

\begin{tabular}{|c|c|c|c|c|c|c|c|c|c|c|}
\hline \multicolumn{11}{|c|}{ Theorem 1(I) If $W_{1} \geq 0$} \\
\hline \multicolumn{11}{|c|}{$t_{d}=0.008, N=0.01$} \\
\hline & $\Delta_{1}$ & $\Delta_{2}$ & $\Delta_{3}$ & $\Delta^{*}$ & $o$ & $h$ & $M$ & $R^{*}$ & $T^{*}$ & $T C\left(T^{*}\right)$ \\
\hline (A) & $<0$ & $<0$ & $<0$ & $<0$ & 0.200 & 1.5 & 0.02 & 0.0237 & $R^{*}=0.0237$ & 1329.5 \\
\hline (B) & $<0$ & $<0$ & $<0$ & $\geq 0$ & 0.150 & 1.5 & 0.02 & 0.0237 & $T_{4}^{*}=0.0218$ & 1327.4 \\
\hline (C) & $<0$ & $<0$ & $\geq 0$ & $\geq 0$ & 0.080 & 1.5 & 0.02 & 0.0237 & $T_{3}^{*}=0.0166$ & 1323.7 \\
\hline (D) & $<0$ & $\geq 0$ & $\geq 0$ & $\geq 0$ & 0.010 & 1.5 & 0.02 & 0.0237 & $T_{2}^{*}=0.0086$ & 1317.9 \\
\hline (E) & $\geq 0$ & $\geq 0$ & $\geq 0$ & $\geq 0$ & 0.005 & 1.5 & 0.02 & 0.0237 & $T_{1}^{*}=0.0072$ & 1317.2 \\
\hline
\end{tabular}

Table 2. The optimal replenishment policy used Theorem 1(II).

\begin{tabular}{rcccccccccccccc}
\hline \multicolumn{110}{c}{ Theorem 1(II) If $\boldsymbol{W}_{\mathbf{1}}<\mathbf{0}$} \\
\hline \multicolumn{110}{c}{$0=0.002, N=0.01, M=0.02$} \\
\hline$\Delta_{1}$ & $\Delta_{2}$ & $\Delta_{3}$ & $\Delta^{*}$ & $h$ & $D$ & $x$ & $p$ & $I_{k}$ & $t_{d}$ & $R^{*}$ & $T^{*}$ & $T C\left(T^{*}\right)$ \\
\hline (A) $<0$ & $\geq 0$ & $\geq 0$ & $\geq 0$ & 0.01 & 95 & 1000 & 0.001 & 0.13 & 0.002 & 0.0210 & $T_{2}^{*}=0.0021$ & 1238.6 \\
\hline (B) $\geq 0$ & $\geq 0$ & $\geq 0$ & $\geq 0$ & 1.50 & 100 & 300 & 0.010 & 0.15 & 0.008 & 0.0237 & $T_{1}^{*}=0.0014$ & 1316.1 \\
\hline
\end{tabular}

Table 3. The optimal replenishment policy used Theorem 2(I).

\begin{tabular}{ccccccccccc}
\hline \multicolumn{10}{c}{ Theorem 2(I) If $\boldsymbol{W}_{\mathbf{2}} \geq \mathbf{0}$} \\
\hline & \multicolumn{8}{c}{$t_{d}=0.012, R^{*}=0.1240$} \\
\hline & $\Delta_{4}$ & $\Delta_{5}$ & $\Delta_{3}$ & $\Delta^{*}$ & $o$ & $h$ & $N$ & $M$ & $T^{*}$ & $T C\left(T^{*}\right)$ \\
\hline (A) & $<0$ & $<0$ & $<0$ & $<0$ & 6.00 & 0.01 & 0.01 & 0.02 & $R^{*}=0.1240$ & 1315.4 \\
\hline (B) & $<0$ & $<0$ & $<0$ & $\geq 0$ & 4.00 & 0.01 & 0.01 & 0.02 & $T_{4}^{*}=0.1190$ & 1299.2 \\
\hline (C) & $<0$ & $<0$ & $<0$ & $\geq 0$ & 0.01 & 0.01 & 0.01 & 0.02 & $T_{3}^{*}=0.0131$ & 1239.5 \\
\hline (D) & $<0$ & $\geq 0$ & $\geq 0$ & $\geq 0$ & 0.01 & 1.50 & 0.01 & 0.02 & $T_{2}^{*}=0.0106$ & 1240.3 \\
\hline (E) & $\geq 0$ & $\geq 0$ & $\geq 0$ & $\geq 0$ & 0.01 & 2.00 & 0.01 & 0.02 & $T_{1}^{*}=0.0094$ & 1240.6 \\
\hline
\end{tabular}

Table 4. The optimal replenishment policy used Theorem 2(II) and (III).

\begin{tabular}{|c|c|c|c|c|c|c|c|c|c|c|c|c|c|}
\hline \multicolumn{14}{|c|}{ Theorem 2(II) If $W_{2}<0$ and $W_{1} \geq 0$} \\
\hline & $\Delta_{4}$ & $\Delta_{5}$ & $\Delta_{3}$ & $\Delta^{*}$ & $o$ & $h$ & $D$ & $c$ & $v$ & $x$ & $I_{k}$ & $I_{e}$ & $t_{d}$ \\
\hline (A) & $<0$ & $<0$ & $\geq 0$ & $\geq 0$ & 0.0005 & 0.0001 & 94 & 0.005 & 3 & 100 & 0.14 & 0.10 & 0.0125 \\
\hline (B) & $<0$ & $\geq 0$ & $\geq 0$ & $\geq 0$ & 0.0020 & 0.0100 & 95 & 3.000 & 4 & 1000 & 0.13 & 0.12 & 0.0120 \\
\hline (C) & $\geq 0$ & $\geq 0$ & $\geq 0$ & $\geq 0$ & 0.0020 & 0.1000 & 95 & 3.000 & 4 & 1000 & 0.13 & 0.12 & 0.0120 \\
\hline \multicolumn{14}{|c|}{ Theorem 2(II) If $W_{2}<0$ and $W_{1} \geq 0$} \\
\hline & $\Delta_{4}$ & $\Delta_{5}$ & $\Delta_{3}$ & $\Delta^{*}$ & \multicolumn{2}{|c|}{$N$} & $M$ & \multicolumn{2}{|c|}{$R^{*}$} & \multicolumn{2}{|c|}{$T^{*}$} & \multicolumn{2}{|c|}{$T C\left(T^{*}\right)$} \\
\hline (A) & $<0$ & $<0$ & $\geq 0$ & $\geq 0$ & \multicolumn{2}{|c|}{0.012} & 0.013 & \multicolumn{2}{|c|}{0.0133} & \multicolumn{2}{|c|}{$T_{3}^{*}=0.0125$} & \multicolumn{2}{|c|}{941.4161} \\
\hline (B) & $<0$ & $\geq 0$ & $\geq 0$ & $\geq 0$ & \multicolumn{2}{|c|}{0.010} & 0.020 & \multicolumn{2}{|c|}{0.1240} & \multicolumn{2}{|c|}{$T_{5}^{*}=0.0104$} & \multicolumn{2}{|c|}{1238.82} \\
\hline (C) & $\geq 0$ & $\geq 0$ & $\geq 0$ & $\geq 0$ & \multicolumn{2}{|c|}{0.010} & 0.020 & \multicolumn{2}{|c|}{0.1240} & \multicolumn{2}{|c|}{$T_{1}^{*}=0.0094$} & \multicolumn{2}{|c|}{1238.9} \\
\hline \multicolumn{14}{|c|}{ Theorem 2(III) If $W_{1}<0$} \\
\hline & $\Delta_{4}$ & $\Delta_{5}$ & $\Delta_{3}$ & $\Delta^{*}$ & $o$ & $h$ & $D$ & $v$ & $x$ & $I_{k}$ & $I_{e}$ & $t_{d}$ & $N$ \\
\hline (A) & $\geq 0$ & $\geq 0$ & $\geq 0$ & $\geq 0$ & 0.0002 & 0.01 & 95 & 4 & 100 & 0.13 & 0.12 & 0.012 & 0.01 \\
\hline \multicolumn{14}{|c|}{ Theorem 2(III) If $W_{1}<0$} \\
\hline & $\Delta_{4}$ & $\Delta_{5}$ & $\Delta_{3}$ & $\Delta^{*}$ & $M$ & $R^{*}$ & \multicolumn{3}{|c|}{$T^{*}$} & \multicolumn{4}{|c|}{$T C\left(T^{*}\right)$} \\
\hline (A) & $\geq 0$ & $\geq 0$ & $\geq 0$ & $\geq 0$ & 0.02 & 0.1240 & \multicolumn{3}{|c|}{$T_{1}^{*}=0.0033$} & \multicolumn{4}{|c|}{1238.6} \\
\hline
\end{tabular}


Table 5. The optimal replenishment policy used Theorem 3(I).

\begin{tabular}{ccccccccccccc}
\hline & \multicolumn{10}{c}{ Theorem 3(I) If $\boldsymbol{W}_{\mathbf{2}} \geq \mathbf{0}$} \\
\hline & $\Delta_{4}$ & $\Delta_{6}$ & $\Delta_{7}$ & $\Delta^{*}$ & $o$ & $h$ & $t_{d}$ & $N$ & $M$ & $R^{*}$ & $T^{*}$ & $T C\left(T^{*}\right)$ \\
\hline (A) & $<0$ & $<0$ & $<0$ & $<0$ & 8 & 1 & 0.025 & 0.01 & 0.02 & 0.0827 & $R^{*}=0.0827$ & 4057.5 \\
\hline (B) & $<0$ & $<0$ & $<0$ & $\geq 0$ & 6 & 1 & 0.025 & 0.01 & 0.02 & 0.0827 & $T_{4}^{*}=0.0795$ & 4033.2 \\
\hline (C) & $<0$ & $<0$ & $\geq 0$ & $\geq 0$ & 0.1 & 1 & 0.025 & 0.01 & 0.02 & 0.0827 & $T_{6}^{*}=0.0225$ & 3919.9 \\
\hline (D) & $<0$ & $\geq 0$ & $\geq 0$ & $\geq 0$ & 0.1 & 2 & 0.025 & 0.01 & 0.02 & 0.0827 & $T_{5}^{*}=0.0168$ & 3922.8 \\
\hline (E) & $\geq 0$ & $\geq 0$ & $\geq 0$ & $\geq 0$ & 0.1 & 8 & 0.025 & 0.01 & 0.02 & 0.0827 & $T_{1}^{*}=0.0089$ & 3933.3 \\
\hline
\end{tabular}

Table 6. The optimal replenishment policy used Theorem 3(II) and (III).

\begin{tabular}{|c|c|c|c|c|c|c|c|c|c|c|c|c|c|}
\hline \multicolumn{14}{|c|}{ Theorem 3 (II) If $W_{2}<0$ and $W_{1} \geq 0$} \\
\hline \multicolumn{14}{|c|}{$t_{d}=0.025$} \\
\hline & $\Delta_{4}$ & $\Delta_{6}$ & $\Delta_{7}$ & $\Delta^{*}$ & $o$ & $h$ & $D$ & $N$ & $N$ & & $R^{*}$ & $T^{*}$ & $T C\left(T^{*}\right)$ \\
\hline (A) & $<0$ & $\geq 0$ & $\geq 0$ & $\geq 0$ & 0.004 & 0.01 & 200 & 0.010 & 0.0 & & 0.1232 & $T_{5}^{*}=0.0102$ & 2608.0 \\
\hline (B) & $\geq 0$ & $\geq 0$ & $\geq 0$ & $\geq 0$ & 0.001 & 0.10 & 300 & 0.010 & 0.0 & & 0.0827 & $T_{1}^{*}=0.0037$ & 3911.4 \\
\hline \multicolumn{14}{|c|}{ Theorem 3(III) If $W_{1}<0$} \\
\hline (A) & $\geq 0$ & $\geq 0$ & $\geq 0$ & $\geq 0$ & 0.001 & 0.01 & 300 & 0.025 & 0.003 & 0.020 & 0.0827 & $T_{1}^{*}=0.0042$ & 3910.8 \\
\hline
\end{tabular}

Table 7. The optimal replenishment policy used Theorem 4(I).

\begin{tabular}{ccccccccccccc}
\hline \multicolumn{10}{c}{ Theorem 4(I) If $\boldsymbol{W}_{\mathbf{3}} \geq \mathbf{0}$} \\
\hline & $\Delta_{8}$ & $\Delta_{9}$ & $\Delta^{* *}$ & $o$ & $h$ & $D$ & $t_{d}$ & $N$ & $M$ & $R^{*}$ & $T^{*}$ & $T C\left(T^{*}\right)$ \\
\hline (A) $\quad<0$ & $<0$ & $<0$ & 2.00 & 1.0 & 200 & 0.01 & 0.03 & 0.02 & 0.0497 & $R^{*}=0.0497$ & 2672.5 \\
\hline (B) $\quad<0$ & $<0$ & $\geq 0$ & 1.00 & 0.5 & 100 & 0.01 & 0.03 & 0.02 & 0.0986 & $T_{9}^{*}=0.0570$ & 1334.7 \\
\hline (C) $\quad<0$ & $\geq 0$ & $\geq 0$ & 0.10 & 1.0 & 200 & 0.01 & 0.03 & 0.02 & 0.0497 & $T_{8}^{*}=0.0151$ & 2618.0 \\
\hline (D) $\geq 0$ & $\geq 0$ & $\geq 0$ & 0.01 & 2.0 & 100 & 0.01 & 0.03 & 0.02 & 0.0986 & $T_{7}^{*}=0.0092$ & 1306.4 \\
\hline
\end{tabular}

Table 8. The optimal replenishment policy used Theorem 4(II).

\begin{tabular}{rcccccccccccc}
\hline \multicolumn{10}{c}{ Theorem 4(II) If $\boldsymbol{W}_{\mathbf{3}}<\mathbf{0}$} \\
\hline$\Delta_{8}$ & $\Delta_{9}$ & $\Delta^{* *}$ & $o$ & $h$ & $D$ & $t_{d}$ & $N$ & $M$ & $R^{*}$ & $T^{*}$ & $T C\left(T^{*}\right)$ \\
\hline (A) $<0$ & $\geq 0$ & $\geq 0$ & 0.01 & 0.10 & 250 & 0.01 & 0.04 & 0.03 & 0.0398 & $T_{8}^{*}=0.0103$ & 3262.4 \\
\hline (B) $\geq 0$ & $\geq 0$ & $\geq 0$ & 0.01 & 2.00 & 250 & 0.01 & 0.04 & 0.03 & 0.0398 & $T_{7}^{*}=0.0058$ & 3264.2 \\
\hline
\end{tabular}

Table 9. The optimal replenishment policy used Theorem 5(I) and 5(II).

\begin{tabular}{cccccccccccccc}
\hline \multicolumn{10}{c}{ Theorem 5(I) If $\boldsymbol{W}_{\mathbf{3}} \geq \mathbf{0}$} \\
\hline & $\Delta_{10}$ & $\Delta_{11}$ & $\Delta^{* *}$ & $o$ & $h$ & $D$ & $t_{d}$ & $N$ & $M$ & $R^{*}$ & $T^{*}$ & $T C\left(T^{*}\right)$ \\
\hline (A) $\quad<0$ & $<0$ & $<0$ & 10 & 1.0 & 300 & 0.03 & 0.04 & 0.02 & 0.0991 & $R^{*}=0.0991$ & 4074.2 \\
\hline (B) & $<0$ & $<0$ & $\geq 0$ & 0.2 & 1.0 & 100 & 0.03 & 0.04 & 0.02 & 0.2878 & $T_{9}^{*}=0.0362$ & 1312.7 \\
\hline (C) & $<0$ & $\geq 0$ & $\geq 0$ & 0.05 & 1.0 & 100 & 0.03 & 0.04 & 0.02 & 0.2878 & $T_{10}^{*}=0.0279$ & 1308.0 \\
\hline (D) & $\geq 0$ & $\geq 0$ & $\geq 0$ & 0.01 & 1.0 & 100 & 0.03 & 0.04 & 0.02 & 0.2878 & $T_{7}^{*}=0.0121$ & 1306.1 \\
\hline \multicolumn{10}{c}{ Theorem 5(II) } & If $\boldsymbol{W}_{\mathbf{3}}<\mathbf{0}$ \\
\hline (A) $\geq 0$ & $\geq 0$ & $\geq 0$ & 0.005 & 1.0 & 300 & 0.03 & 0.04 & 0.02 & 0.0991 & $T_{7}^{*}=0.0049$ & 3915.4 \\
\hline
\end{tabular}

Example 1. $h=1.5, c=3, v=4, D=100, x=300, p=0.01, s=10, t_{d}=0.008$, $N=0.01$ year, $M=0.02$ year, $L=0.3, I_{k}=\$ 0.15 / \$ /$ year, $I_{e}=\$ 0.12 / \$ /$ year, $\tau=0.3, \alpha=0.3$, 
$\beta=0.4$ and $\rho=0.3$, Theorem 1(I) (if $W_{1} \geq 0$ ) is applied to obtain optimal solution The computed result is shown in Table 1.

Example 2. $o=0.0002, c=3, v=4, s=10, N=0.01$ year, $M=0.02$ year, $L=0.3$, $I_{e}=\$ 0.12 / \$ /$ year, $\tau=0.3, \alpha=0.3, \beta=0.4$ and $\rho=0.3$, Theorem 1(II) (if $W_{1}<0$ ) is applied to obtain optimal solution. The computed result is shown in Table 2.

Example 3. $c=3, v=4, D=95, x=300, p=0.01, s=10, t_{d}=0.012, N=0.01$ year, $M=0.02$ year, $L=0.3, I_{k}=\$ 0.15 / \$ /$ year, $I_{e}=\$ 0.12 / \$ /$ year, $\tau=0.3, \alpha=0.3, \beta=0.4$ and $\rho=0.3$, Theorem 2(I) (if $W_{2} \geq 0$ ) is applied to obtain optimal solution. The computed result is shown in Table 3.

Example 4. $p=0.01, s=10, L=0.3, \tau=0.3, \alpha=0.3, \beta=0.4$ and $\rho=0.3$, Theorem 2(II) (if $W_{2}<0$ and $W_{1} \geq 0$ ) and Theorem 2(III) (if $W_{1}<0$ ) are applied to obtain optimal solution. The computed result is shown in Table 4 .

Example 5. $c=3, v=4, D=300, x=1000, p=0.001, s=10, t_{d}=0.025, N=0.01$ year, $M=0.02$ year, $L=0.3, I_{k}=\$ 0.13 / \$ /$ year, $I_{e}=\$ 0.12 / \$ /$ year, $\tau=0.3, \alpha=0.3, \beta=0.4$ and $\rho=0.3$, Theorem 3(I) (if $W_{2} \geq 0$ ) is applied to obtain optimal solution. The computed result is shown in Table 5.

Example 6. $c=3, v=4, x=1000, p=0.001, s=10, t_{d}=0.025, M=0.02$ year, $L=0.3$, $I_{k}=\$ 0.13 / \$ /$ year, $I_{e}=\$ 0.12 / \$ /$ year, $\tau=0.3, \alpha=0.3, \beta=0.4$ and $\rho=0.3$, Theorem 3(II) (if $W_{2}<0$ and $W_{1} \geq 0$ ) and Theorem 3(III) (if $W_{1}<0$ ) are applied to obtain optimal solution. The computed result is shown in Table 6.

Example 7. $c=3, v=4, x=1000, p=0.001, s=10, t_{d}=0.01, N=0.03$ year, $M=0.02$ year, $L=0.3, I_{k}=\$ 0.13 / \$ /$ year, $I_{e}=\$ 0.12 / \$ /$ year, $\tau=0.3, \alpha=0.3, \beta=0.4$ and $\rho=0.3$, Theorem 4(I) (if $W_{3} \geq 0$ ) is applied to obtain optimal solution. The computed result is shown in Table 7 .

Example 8. $o=0.01, c=3, v=4, D=250, x=1000, p=0.001, s=10, t_{d}=0.01$, $N=0.04$ year, $M=0.03$ year, $L=0.3, I_{k}=\$ 0.13 / \$ /$ year, $I_{e}=\$ 0.12 / \$ /$ year, $\tau=0.3, \alpha=0.3$, $\beta=0.4$ and $\rho=0.3$, Theorem 4(II) (if $W_{3}<0$ ) is applied to obtain optimal solution. The computed result is shown in Table 8 .

Example 9. $h=1, c=3, v=4, D=250, x=1000, p=0.001, s=10,=0.03, N=0.04$ year, $M=0.02$ year, $L=0.3, I_{k}=\$ 0.13 / \$ /$ year, $I_{e}=\$ 0.12 / \$ /$ year, $\tau=0.3, \alpha=0.3, \beta=0.4$ and $\rho=0.3$, Theorem 5(I) (if $W_{3} \geq 0$ ) and Theorem 5(II) (if $W_{3}<0$ ) are applied to obtain optimal solution. The computed result is shown in Table 9.

Additionally, in Tables 1-9, $R^{*}$ and $\Delta^{*}$ is defined as Equations (11) and (56), respectively. $T^{*}$ is the optimal cycle time so that $T C\left(T^{*}\right)$ is the minimum.

\section{Conclusions and Potential Directions for Further Research}

In our present investigation, we have established a sustainable inventory system in which the retailer sells the non-instantaneous deteriorating item that is fully deteriorated close to its expiry date and has imperfect quality such as those in seasonal products, food products, electronic components, and others. In order to manage the quality of the items, an inspection will occur during the state in which there is no deterioration. On the other hand, the supplier demands the retailer a distinct payment scheme, such as partial prepayment or cash and trade credit; in turn, the retailer grants customers partial cash and trade credit.

We have observed that some of the optimization methods lack mathematical rigor, and some of them are based on intuitive arguments, which result in the solution procedures being questionable from the logical viewpoints of mathematical analysis, such as those in the earlier works by Chang et al. (2004), Ouyang et al. (2006), and Cheng and Wang (2009). They ignored explorations of interrelations of functional behaviors of the total cost function to locate the optimal solution, so those shortcomings will naturally influence the implementation of their considered inventory model. Essentially, in order to explore the functional behaviors (such as continuity, monotonicity (increasing and decreasing) properties, differentiability, etc.) of the object functions (that is, the total cost functions), one can and should apply the mathematically accurate and reliable solution procedures. Moreover, if the object function (that is, the total cost functions) are convex, it is easier to find the optimal solution by using the convexity property. Consequently, the discussion 
of the convexity of the total annual cost function is one of the main research topics of this article.

The object function (that is, the total cost function) of this article is a piecewise continuous function; it is increasing or decreasing in its own domain and we discuss the continuity of the object function, especially at its extreme point(s). Furthermore, the main purpose of this article has been to provide accurate and reliable mathematical analytic solution procedures for different scenarios by studying the convexity of the total annual cost function and the functional behaviors of the object function. For the proposed models, the convexity of the object functions has been proved and the closed-form optimal solution has been derived. Numerical examples, which illustrate the behavior of proposed models and the applied solution method, have been considered; a retailer, using the model obtained in this article, can effectively determine the optimal replenishment cycle.

Finally, the limitation of this article is that we have concentrated upon the inventory system without shortage, which can affect the supply chain from the producer to the retailer. Furthermore, this model has the potential to be extended to incorporate inflation and quantity discount effects, different demand forms such as credit-linked promotiondependent demand, and other issues under the system with shortages. Additionally, this article has considered the deterministic situation, so considering the stochastic situation, such as stochastic demand, can be another future research direction on the subject of this article.

Author Contributions: Conceptualization, J.-J.L., S.-D.L. and K.-J.C.; methodology, J.-J.L., S.-D.L. and H.M.S.; software, J.-J.L. and K.-N.H.; validation, J.-J.L., S.-D.L. and H.M.S.; formal analysis, J.-J.L., S.-D.L. and H.M.S.; investigation, J.-J.L., K.-N.H. and S.-D.L.; resources, J.-J.L., K.-J.C. and S.-D.L.; data curation, K.-N.H., S.-D.L. and S.-F.L.; writing-original draft preparation, J.-J.L., K.-J.C., S.-D.L. and S.-F.L.; writing-review and editing, J.-J.L. and S.-D.L.; visualization, J.-J.L. and S.-F.L.; supervision, J.-J.L., S.-D.L. and H.M.S.; project administration, J.-J.L., K.-J.C., S.-D.L. and H.M.S.; funding acquisition, Not Applicable. All authors have read and agreed to the published version of the manuscript.

Funding: This research received no external funding.

Institutional Review Board Statement: Not applicable.

Informed Consent Statement: Not applicable.

Data Availability Statement: Not applicable.

Conflicts of Interest: The authors declare no conflict of interest.

\section{Notations}

If and where needed, additional notations will be introduced throughout the paper.

o

$h$

$y$

c

$v$

D

$x$ the ordering cost in dollars per order; unit stock holding cost, excluding interest charges (\$/unit/year); the order quantity in units; unit purchasing cost (\$/unit); unit selling price (\$/unit), $(v>c)$; the market annual demand rate in units; the screening rate, $(x>D)$; the percentage of defective items in $y$; the screening cost per unit; the expiration date or the maximum lifetime of the deteriorating item in years, $(0<m<5)$; 


\begin{tabular}{|c|c|}
\hline$\theta(t)$ & $\begin{array}{l}\text { the time-varying deterioration rare at time } t \text {, where } \\
0 \leq \theta(t) \leq 1\end{array}$ \\
\hline$t_{d}$ & the fresh product time; \\
\hline$t_{s}$ & the screening time per cycle; \\
\hline M & the upstream credit period by the supplier to the retailer; \\
\hline$N$ & the downstream credit period by the retailer to customers; \\
\hline$I_{e}$ & the interest earned per dollar per year; \\
\hline$I_{k}$ & the interest charged by the supplier per dollar per year; \\
\hline$L$ & $\begin{array}{l}\text { the length of time in years during which the prepayments } \\
\text { are paid; }\end{array}$ \\
\hline$\alpha$ & $\begin{array}{l}\text { the fraction of procurement cost to be prepaid before the } \\
\text { time of delivery, } 0 \leq \alpha \leq 1 \text {; }\end{array}$ \\
\hline$\beta$ & $\begin{array}{l}\text { the fraction of procurement cost to be paid at the time of } \\
\text { delivery, } 0 \leq \beta \leq 1\end{array}$ \\
\hline$\tau$ & $\begin{array}{l}\text { the fraction of procurement cost granted a credit period } \\
\text { from the supplier to the retailer, } 0 \leq \tau \leq 1 \text { and } \\
\alpha+\beta+\tau=1\end{array}$ \\
\hline$\rho$ & $\begin{array}{l}\text { the fraction of the sales revenue offered a credit period by } \\
\text { the retailer to the customers, } 0 \leq \rho \leq 1 \text {; }\end{array}$ \\
\hline$T C(T)$ & the total annual relevant cost in dollars; \\
\hline$T$ & the length of inventory cycle time in years, $T \leq m$; \\
\hline$T^{*}$ & the (fixed) optimal cycle time of $T C(T)$; \\
\hline$I_{1}(t)$ & $\begin{array}{l}\text { the inventory level at time } t \in\left[0, t_{s}\right] \text { in which the product } \\
\text { has no deterioration; }\end{array}$ \\
\hline$I_{2}(t)$ & $\begin{array}{l}\text { the inventory level at time } t \in\left[t_{s}, t_{d}\right] \text { in which the product } \\
\text { has no deterioration; }\end{array}$ \\
\hline$I_{3}(t)$ & $\begin{array}{l}\text { the inventory level at time } t \in\left[t_{d}, T\right] \text { in which the product } \\
\text { has deterioration; }\end{array}$ \\
\hline
\end{tabular}

\section{Assumptions}

1. All deteriorating items continuously deteriorate with time and cannot be sold when time exceeds the expiration date $m$. To make the problem tractable, we assume the same as in Wang et al. [65] and Chen et al. [16], that the deterioration rate is $\theta(t)=\frac{1}{1+m-t}, 0 \leq t \leq T \leq m$.

2. There is no replacement or repair of deteriorated items during the replenishment cycle time $(0, T]$.

3. The demand rate is known and constant.

4. Shortages are not allowed.

5. The replenishment rate is infinite.

6. The time horizon is infinite.

7. There exists an inspection process that is $100 \%$ effective.

8. The screening rate is faster than the demand rate.

9. The supplier imposes a prepayment policy to the retailer, in which the retailer should prepay a fraction of procurement cost ( $\alpha$ percent) at the moment they place an order to the supplier (at time $L$ ), they pay another $\beta$ percentage of procurement cost at time 0 upon the receipt of all items, and receive an upstream credit period of $M$ years on the remaining $\tau$ portion of procurement cost.

10. During the selling period, the retailer offers the partial trade credit to his customers, in which their customers must immediately make a partial payment (at the rate $1-\rho$ ) to the retailer in cash at the time of purchasing items and then receive credit period $N$ on the outstanding amount.

11. If $M \geq N$, then the retailer deposits the sales revenue into an interest bearing account. If $M \geq T+N$ (i.e., the permissible delay period is longer than the time at which the retailer receives the last payment from its customers), then the retailer receives all revenue and pays off the entire purchase cost at the end of the permissible delay $M$. Otherwise, (if $M \leq T+N$ ), the retailer pays the supplier the sum of all units sold by $M-N$ and the collateral deposit received from $N$ to $M$, keeps the profit for the use of the other activities, and starts paying for the interest charges on the items sold after $M-N$. 
12. If $N \geq M$, then the retailer finances and pays its supplier the entire amount of the delayed payment $(1-\rho) c D T$ at the end of the trade credit $M$, and then pays down the loan after time $N$ at which the retailer starts to receive sales revenue from its customers. For the collateral deposit the retailer deposits the sales revenue into an interest bearing account until the end of the permissible delay $M$. If $T \geq M$, then the retailer pays the supplier all units sold by $M$, keeps the profit for the use of the other activities, and starts paying for the interest charges on the items sold after $M$.

\section{Appendix A}

Proof of Lemma 2. In proving Lemma 2, we consider the assertions of Lemma 2 item-wise.

(A) Taking the first-order derivative of $G(T)$ with respect to $T$, we obtain

$$
\begin{aligned}
G^{\prime}(T) & =\frac{D\left(1+m-t_{d}\right) t_{d} T}{(1+m-T)^{2}}+\frac{2 p D^{2}}{x(1-p)^{2}}\left\{\left[t_{d}+\left(1+m-t_{d}\right)\right.\right. \\
& \left.\left.\ln \left(\frac{1+m-t_{d}}{1+m-T}\right)\right] \cdot \frac{\left(1+m-t_{d}\right) T}{(1+m-T)^{2}}+\frac{\left(1+m-t_{d}\right)^{2} T}{(1+m-T)^{2}}\right\} \\
& +\frac{D}{2}\left(1+m-t_{d}\right)^{2} \cdot \frac{T}{(1+m-T)^{2}}+\frac{D T}{2} \\
& >0
\end{aligned}
$$

and

$$
G\left(t_{d}\right)=\frac{p D^{2}}{x(1-p)^{2}}>0
$$

Furthermore, we see that $G(T)>0$ if $T \geq t_{d}$.

(B) We define $g(T)$ as follows:

$$
g(T)=D\left(1+m-t_{d}\right) \frac{T}{1+m-T}-D t_{d}-D\left(1+m-t_{d}\right) \cdot \ln \left(\frac{1+m-t_{d}}{1+m-T}\right)
$$

Taking the first-order derivative of $g(T)$ with respect to $T$, we derive

$$
g^{\prime}(T)=\frac{D\left(1+m-t_{d}\right) T}{(1+m-T)^{2}}>0
$$

and

$$
g\left(t_{d}\right)=0
$$

Furthermore, we have

$$
D\left(1+m-t_{d}\right) \frac{T}{1+m-T}-D t_{d}-D\left(1+m-t_{d}\right) \ln \left(\frac{1+m-t_{d}}{1+m-T}\right)>0 \text { if } T \geq t_{d}
$$

(C) Taking the first-order derivative of $H(T)$ with respect to $T$, we obtain

$$
\begin{aligned}
H^{\prime}(T) & =\frac{4 p D^{2}}{x(1-p)^{2}}\left(1+m-t_{d}\right)\left[t_{d}+\left(1+m-t_{d}\right) \cdot \ln \left(\frac{1+m-t_{d}}{1+m-T}\right)\right] \frac{T^{2}}{(1+m-T)^{3}} \\
& +\frac{6 p D^{2}}{x(1-p)^{2}}\left(1+m-t_{d}\right)^{2} \frac{T^{2}}{(1+m-T)^{3}}
\end{aligned}
$$

and

$$
H\left(t_{d}\right)=\frac{D t_{d}^{3}}{\left(1+m-t_{d}\right)}+\frac{2 p D^{2}}{x(1-p)^{2}} \cdot \frac{t_{d}^{3}}{\left(1+m-t_{d}\right)}>0
$$

Furthermore, we have $H(T)>0$ if $T \geq t_{d}$.

(D) We define $h(T)$ by

$$
\begin{aligned}
h(T) & =D\left(1+m-t_{d}\right) \frac{(1+m) T}{\left(1+m-T^{2}\right.}-3 D\left(1+m-t_{d}\right) \frac{T}{1+m-T}+2 D t_{d} \\
& +2 D\left(1+m-t_{d}\right) \cdot \ln \left(\frac{1+m-t_{d}}{1+m-T}\right)
\end{aligned}
$$


Taking the first-order derivative of $h(T)$ with respect to $T$, we find that

$$
h^{\prime}(T)=D\left(1+m-t_{d}\right) \frac{2 T^{2}}{(1+m-T)^{3}}>0
$$

and

$$
h\left(t_{d}\right)=\frac{D t_{d}^{2}}{\left(1+m-t_{d}\right)}>0
$$

So, we finally have

$$
\begin{aligned}
& D\left(1+m-t_{d}\right) \frac{(1+m) T}{(1+m-T)^{2}}-3 D\left(1+m-t_{d}\right) \frac{T}{1+m-T} \\
& +2 D t_{d}+2 D\left(1+m-t_{d}\right) \cdot \ln \left(\frac{1+m-t_{d}}{1+m-T}\right)>0
\end{aligned}
$$

if $T \geq t_{d}$.

We thus have completed the proof of Lemma 2 .

\section{References}

1. Zhang, A.X. Optimal advance payment scheme involving fixed per-payment costs. Omega 1996, 24, 577-582. [CrossRef]

2. Taleizadeh, A.A.; Pentico, D.W.; Jabalameli, M.S.; Aryanezhad, M. An economic order quantity model with multiple partial prepayments and partial backordering. Math. Comput. Model. 2013, 57, 311-323. [CrossRef]

3. Taleizadeh, A.A. An economic order quantity model for deteriorating item in a purchasing system with multiple prepayments. Appl. Math. Model. 2014, 38, 5357-5366. [CrossRef]

4. Taleizadeh, A.A. An EOQ model with partial backordering and advance payments for an evaporating item. Int. J. Prod. Econ. 2014, 155, 185-193. [CrossRef]

5. Zhang, Q.; Tsao, Y.-C.; Chen, T.-H. Economic order quantity under advance payment. Appl. Math. Model. 2014, 38, 5910-5921. [CrossRef]

6. Eck, K.; Engemann, M.; Schnitzer, M. How trade credits foster exporting. Rev. World Econ. 2014, 151, 73-101. [CrossRef]

7. Lashgari, M.; Taleizadeh, A.A.; Ahmadi, A. Partial up-stream advanced payment and partial down-stream delayed payment in a three-level supply chain. Ann. Oper. Res. 2016, 238, 329-354. [CrossRef]

8. Tavakoli, S.; Taleizadeh, A.A. An EOQ model for decaying item with full advanced payment and conditional dis-count. Ann. Oper. Res. 2017, 259, 415-436. [CrossRef]

9. Heydari, J.; Rastegar, M.; Glock, C. A two-level delay in payments contract for supply chain coordination: The case of creditdependent demand. Int. J. Prod. Econ. 2017, 191, 26-36. [CrossRef]

10. Feng, L.; Chan, Y.-L. Joint pricing and production decisions for new products with learning curve effects under up-stream and downstream trade credits. Eur. J. Oper. Res. 2019, 272, 905-913. [CrossRef]

11. Chen, S.-C.; Teng, J.-T. Inventory and credit decisions for time-varying deteriorating items with up-stream and down-stream trade credit financing by discounted cash flow analysis. Eur. J. Oper. Res. 2015, 243, 566-575. [CrossRef]

12. Mahata, G.C. Optimal ordering policy with trade credit and variable deterioration for fixed life time products. Int. J. Oper. Res. 2016, 25, 307-326. [CrossRef]

13. $\mathrm{Wu}, \mathrm{J}$; Teng, J.-T.; Chan, Y.-L. Inventory policies for perishable products with expiration dates and ad-vance-cash-credit payment schemes. Int. J. Syst. Sci. Oper. Logist. 2018, 5, 310-326.

14. Zia, N.P.; Taleizadeh, A.A. A lot-sizing model with backordering under hybrid linked-to-order multiple advance payments and delayed payment. Transp. Res. Part E Logist. Transp. Rev. 2015, 82, 19-37. [CrossRef]

15. Wu, J.; Al-Khateeb, F.B.; Teng, J.-T.; Cárdenas-Barrón, L.E. Inventory models for deteriorating items with maximum lifetime under downstream partial trade credits to credit-risk customers by discounted cash-flow analysis. Int. J. Prod. Econ. 2016, 171, 105-115. [CrossRef]

16. Chen, S.-C.; Min, J.; Teng, J.-T.; Li, F. Inventory and shelf-space management for fresh produce with fresh-ness-and-stock dependent demand and expiration date. J. Oper. Res. Soc. 2016, 67, 884-896. [CrossRef]

17. Teng, J.-T.; Cárdenas-Barrón, L.E.; Chang, H.-J.; Wu, J.; Hu, Y. Inventory lot-size policies for deteriorating items with expiration dates and advance payments. Appl. Math. Model. 2016, 40, 8605-8616. [CrossRef]

18. Diabat, A.; Taleizadeh, A.A.; Lashgari, M. A lot sizing model with partial downstream delayed payment, partial upstream advance payment, and partial backordering for deteriorating items. J. Manuf. Syst. 2017, 45, 322-342. [CrossRef]

19. Feng, L.; Chan, Y.-L.; Cárdenas-Barrón, L.E. Pricing and lot-sizing polices for perishable goods when the demand depends on selling price, displayed stocks, and expiration date. Int. J. Prod. Econ. 2017, 185, 11-20. [CrossRef]

20. Mahata, G.C.; De, S.K. Supply chain inventory model for deteriorating items with maximum lifetime and partial trade credit to credit-risk customers. Int. J. Manag. Sci. Eng. Manag. 2017, 12, 21-32. [CrossRef]

21. Tiwari, S.; Cárdenas-Barrón, L.E.; Goh, M.; Shaikh, A.A. Joint pricing and inventory model for deteriorating items with expiration dates and partial backlogging under two-level partial trade credits in supply chain. Int. J. Prod. Econ. 2018, 200, 16-36. [CrossRef] 
22. Taleizadeh, A.A.; Akram, R.; Lashgari, M.; Heydari, J. A three-level supply chain with up-stream and down-stream trade credit Periods linked to ordered quantity. Appl. Math. Model. 2017, 40, 8777-8793. [CrossRef]

23. Li, R.; Chan, Y.-L.; Chang, C.-T.; Cárdenas-Barrón, L.E. Pricing and lot-sizing policies for perishable products with advance-cashcredit payments by a discounted cash-flow analysis. Int. J. Prod. Econ. 2017, 193, 578-589. [CrossRef]

24. Li, R.; Liu, Y.; Teng, J.-T.; Tsao, Y.-C. Optimal pricing, lot-sizing and backordering decisions when a seller demands an advancecash-credit payment scheme. Eur. J. Oper. Res. 2019, 278, 283-295. [CrossRef]

25. Taleizadeh, A.A. Lot-sizing model with advance payment pricing and disruption in supply under planned partial backordering. Int. Trans. Oper. Res. 2017, 24, 783-800. [CrossRef]

26. Krommyda, I.-P.; Skouri, K.; Lagodimos, A. A unified EOQ model with financial constraints and market tolerance. Appl. Math. Model. 2019, 65, 89-105. [CrossRef]

27. Tsao, Y.-C.; Putri, R.P.F.R.; Zhang, C.; Linh, V.T. Optimal pricing and ordering policies for perishable products under advancecash-credit payment scheme. J. Ind. Eng. Int. 2019, 15, 131-146. [CrossRef]

28. Mashud, A.H.M.; Roy, D.; Daryanto, Y.; Wee, H.-M. A Joint pricing deteriorating inventory model considering the product life cycle and advance payment. RAIRO-Oper. Res. 2021, 55, S1069-S1088. [CrossRef]

29. Mashud, A.H.M.; Roy, D.; Daryanto, Y.; Chakraborty, R.K.; Tseng, M.-L. A controllable carbon emission and deteri-oration inventory model with advance payments scheme. J. Clean. Prod. 2021, 296, 126608. [CrossRef]

30. Arjani, A.; Miah, M.M.; Uddin, M.S.; Mashud, A.H.M.; Wee, H.-M.; Sana, S.S.; Srivastava, H.M. A sustainable economic recycle quantity model for imperfect production system with shortages. J. Risk Financ. Manag. 2021, 14, 173. [CrossRef]

31. Hou, K.-L.; Srivastava, H.M.; Lin, L.-C.; Lee, S.-F. The impact of system deterioration and product warranty on optimal lot sizing with maintenance and shortages backordered. Rev. Real Acad. Cienc. Exactas Físicas Nat. Ser. A Mat. 2021, 115, 1-18. [CrossRef]

32. Udayakumar, R.; Geetha, K.V. Economic ordering policy of single item inventory mode over finite time horizon. Int. J. Syst. Assur. Eng. Manag. 2017, 8, 734-757. [CrossRef]

33. Lashgari, M.; Taleizadeh, A.A.; Sadjadi, S.J. Ordering policies for non-instantaneous deteriorating items under hybrid partial prepayment, partial trade credit and partial backordering. J. Oper. Res. Soc. 2017, 69, 1167-1196. [CrossRef]

34. Udayakumar, R.; Geetha, K.V. An EOQ model for non-instantaneous deteriorating items with two levels of storage under trade credit policy. J. Ind. Eng. Int. 2018, 14, 343-365. [CrossRef]

35. Babangida, B.; Baraya, Y. An inventory model for non-instantaneous deteriorating items with time dependent quadratic demand, two storage facilities and shortages under trade credit policy. Int. J. Model. Oper. Manag. 2020, 8, 1-44. [CrossRef]

36. Soni, N.H.; Suthar, D.N. Optimal pricing and replenishment policy for non-instantaneous deteriorating items with varying rate of demand and partial backlogging. Opserch 2020, 57, 986-1021. [CrossRef]

37. Çalışkan, C. An Inventory Ordering Model for Deteriorating Items with Compounding and Backordering. Symmetry 2021, 13, 1078. [CrossRef]

38. Tiwari, S.; Cárdenas-Barrón, L.E.; Khanna, A.; Jaggi, C.K. Impact of trade credit and inflation on retailer's ordering policies for non-instantaneous deteriorating items in a two-warehouse environment. Int. J. Prod. Econ. 2016, 176, 154-169. [CrossRef]

39. Tsao, Y.-C.; Zhang, Q.; Chang, F.-C.; Vu-Thuy, L. An imperfect production model under Radio Frequency Identification adoption and trade credit. Appl. Math. Model. 2016, 42, 493-508. [CrossRef]

40. Geetha, K.V.; Udayakumar, R. Optimal lot sizing policy for non-instantaneous deteriorating items with price and ad-vertisement dependent demand under partial backlogging. Int. J. Appl. Comput. Math. 2016, 2, 171-193. [CrossRef]

41. Jaggi, C.K.; Tiwari, S.; Goel, S.K. Credit financing in economic ordering policies for non-instantaneous deteriorating items with price dependent demand and two storage facilities. Ann. Oper. Res. 2017, 248, 253-280. [CrossRef]

42. Maihami, R.; Karimi, B.; Ghomi, S.F. Effect of two-echelon trade credit on pricing-inventory policy of non-instantaneous deteriorating products with probabilistic demand and deterioration functions. Ann. Oper. Res. 2017, 257, 237-273. [CrossRef]

43. Mashud, A.; Khan, M.; Uddin, M.; Islam, M. A non-instantaneous inventory model having different deterioration rates with stock and price dependent demand under partially backlogged shortages. Uncertain Supply Chain. Manag. 2018, 6, 49-64. [CrossRef]

44. Bounkhel, M.; Tadj, L.; Benhadid, Y.; Hedjar, R. Optimal Control of Nonsmooth Production Systems with Deteriorating Items, Stock-Dependent Demand, with or without Backorders. Symmetry 2019, 11, 183. [CrossRef]

45. Udayakumar, R.; Geetha, K.; Sana, S.S. Economic ordering policy for non-instantaneous deteriorating items with price and advertisement dependent demand and permissible delay in payment under inflation. Math. Methods Appl. Sci. 2021, 44, 7697-7721. [CrossRef]

46. Khanna, A.; Kishore, A.; Jaggi, C.K. Strategic production modeling for defective items with imperfect inspection pro-cess, rework, and sales return under two-level trade credit. Int. J. Ind. Eng. Comput. 2016, 8, 85-118.

47. Zhou, Y.; Chen, C.; Li, C.; Zhong, Y. A synergic economic order quantity model with trade credit, shortages, imperfect quality and inspection errors. Appl. Math. Model. 2016, 40, 1012-1028. [CrossRef]

48. Datta, T.K. Inventory system with defective products and investment opportunity for reducing defective proportion. Oper. Res. 2017, 17, 297-312. [CrossRef]

49. Taleizadeh, A.A.; Lashgari, M.; Akram, R.; Heydari, J. Imperfect economic production quantity model with upstream trade credit periods linked to raw material order quantity and downstream trade credit periods. Appl. Math. Model. 2016, 40, 8777-8793. [CrossRef] 
50. Pal, S.; Mahapatra, G.S. A manufacturing-oriented supply chain model for imperfect quality with inspection errors, stochastic demand under rework and shortages. Comput. Ind. Eng. 2017, 106, 299-314. [CrossRef]

51. Khakzad, A.; Gholamian, M.R. The effect of inspection on deterioration rate: An inventory model for deteriorating items with advanced payment. J. Clean. Prod. 2020, 254, 120117. [CrossRef]

52. Taleizadeh, A.A.; Khanbaglo, M.P.S.; Cárdenas-Barrón, L.E. Replenishment of imperfect items in an EOQ inventory model with partial backordering. RAIRO-Oper. Res. 2020, 54, 413-434. [CrossRef]

53. Wang, W.T.; Wee, H.M.; Cheng, Y.L.; Wen, C.L.; Cárdenas-Barrón, L.E. EOQ model for imperfect quality items with partial backorders and screening constraint. Eur. J. Ind. Eng. 2015, 9, 744. [CrossRef]

54. Alamri, A.A.; Irina, H.; Aris, A.S. Efficient inventory control for imperfect quality items. Eur. J. Oper. Res. 2016, 254, 92-104. [CrossRef]

55. Palanivel, M.; Uthayakumar, R. Inventory model with imperfect items, stock dependent demand and permissible delay in payments under inflation. RAIRO-Oper. Res. 2016, 50, 473-489. [CrossRef]

56. Aghili, S.J.; Hoseinabadi, H.H. Reliability evaluation of repairable systems using various fuzzy-based methods-a sub-station automation case study. Int. J. Electr. Power Energy Syst. 2017, 85, 130-142. [CrossRef]

57. Tsao, Y.-C. Joint location, inventory, and preservation decisions for non-instantaneous deterioration items under delay in payments. Int. J. Syst. Sci. 2014, 47, 572-585. [CrossRef]

58. Tsao, Y.-C.; Lee, P.-L.; Liao, L.-W.; Zhang, Q.; Vu, T.-L.; Tsai, J. Imperfect economic production quantity models under predictive maintenance and reworking. Int. J. Syst. Sci. Oper. Logist. 2020, 7, 347-360. [CrossRef]

59. Khanna, A.; Mittal, M.; Gautam, P.; Jaggi, C.K. Credit financing for deteriorating imperfect quality items with allowable shortages. Decis. Sci. Lett. 2016, 5, 45-60. [CrossRef]

60. Liao, J.-J.; Huang, K.; Chung, K.; Lin, S.; Ting, P.; Srivastava, H.M. Retailer's optimal ordering policy in the EOQ model with imperfect-quality items under limited storage capacity and permissible delay. Math. Methods Appl. Sci. 2018, 41, 7624-7640. [CrossRef]

61. Kazemi, N.; Abdul-Rashid, S.H.; Ghazilla, R.A.R.; Shekarian, E.; Zanoni, S. Economic order quantity models for items with imperfect quality and emission considerations. Int. J. Syst. Sci. Oper. Logist. 2018, 5, 99-115. [CrossRef]

62. Liao, J.-J.; Huang, K.-N.; Chung, K.-J.; Lin, S.-D.; Chuang, S.-T.; Srivastava, H.M. Optimal ordering policy in an economic order quantity (EOQ) model for non-instantaneous deteriorating items with defective quality and permissible delay in payments. Rev. Real Acad. Cienc. Exactas Físicas Nat. Ser. A Mat. 2020, 114, 41. [CrossRef]

63. Mashud, A.H.M.; Roy, D.; Daryanto, Y.; Ali, M.H. A Sustainable Inventory Model with Imperfect Products, Deterioration, and Controllable Emissions. Mathematics 2020, 8, 2049. [CrossRef]

64. Srivastava, H.M.; Chung, K.-J.; Liao, J.-J.; Lin, S.-D.; Lee, S.-F. An accurate and reliable mathematical analytic solution procedure for the EOQ model with non-instantaneous receipt under supplier credits. Rev. Real Acad. Cienc. Exactas Físicas Nat. Ser. A Mat. 2020, 115, 1-22. [CrossRef]

65. Wang, W.-C.; Teng, J.-T.; Lou, K.-R. Seller's optimal credit period and cycle time in a supply chain for deteriorating items with maximum lifetime. Eur. J. Oper. Res. 2014, 232, 315-321. [CrossRef] 